WC - March 23, 2022

\title{
Variational quantum Monte Carlo study of two-dimensional Wigner crystals: exchange, correlation, and magnetic field effects
}

\author{
Xuejun Zhu ${ }^{1,2}$ and Steven G. Louie ${ }^{2}$ \\ ${ }^{1}$ Department of Physics \& Astronomy, Rutgers University, Piscataway, NJ 08855 \\ ${ }^{2}$ Department of Physics, University of California, and, \\ Materials Sciences Division, Lawrence Berkeley Laboratory, Berkeley, CA 94720
}

(March 23, 2022)

\begin{abstract}
The two-dimensional Wigner crystals are studied with the variational quantum Monte Carlo method. The close relationship between the groundstate wavefunction and the collective excitations in the system is illustrated, and used to guide the construction of the ground-state wavefunction of the strongly correlated solid. Exchange, correlation, and magnetic field effects all give rise to distinct physical phenomena. In the absence of any external magnetic field, interesting spin-orderings are observed in the ground-state of the electron crystal in various two-dimensional lattices. In particular, twodimensional bipartite lattices are shown not to lead necessarily to an antiferromagnetic ground-state. In the quantum Hall effect regime, a strong magnetic field introduces new energy and length scales. The magnetic field quenches the kinetic energy and poses constraints on how the electrons may correlate with each other. Care is taken to ensure the appropriate translational prop-
\end{abstract}


erties of the wavefunction when the system is in a uniform magnetic field. We have examined the exchange, intra-Landau-level correlation as well as Landau-level-mixing effects with various variational wavefunctions. We also determine their dependences on the experimental parameters such as the carrier effective mass at a modulation-doped semiconductor heterojunction. Our results, when combined with some recent calculations for the energy of the fractional quantum Hall liquid including Landau-level-mixing, show quantitatively that in going from $n$-doping to $p$-doping in GaAS/AlGaAS heterojunction systems, the crossover filling factor from the fractional quantum Hall liquid to the Wigner crystal changes from filling factor $\nu \sim 1 / 5$ to $\nu \sim 1 / 3$. This lends strong support to the claim that the observed reentrant insulating phases around $\nu=1 / 5$ for $n$-doped and around $\nu=1 / 3$ for $p$-doped highmobility samples are primarily caused by electron-electron interaction effects. We discuss the possible implications of our theoretical results for some recent experiments carried out in the quantum Hall regime in search of the electron solid.

PACS: 71.45.Nt, 73.40.Kp 


\section{INTRODUCTION}

In this paper, we present a comprehensive study of the various aspects, mostly related to the ground-state energies, of the properties of the two-dimensional (2D) electron Wigner crystal (WC) [1]. Numerical calculations are carried out with the variational quantum Monte Carlo (VMC) method. This approach, pioneered by McMillan for ${ }^{4} \mathrm{He}$ systems [2], has been used extensively to study many fermion systems [3] 7]. The purpose of the present work is two-fold. One is to examine how to construct a good variational ground-state wavefunction for the electron solid by exploring the intimate relation between the groundstate wavefunction and the collective excitation properties. This construction is followed both in the presence and in the absence of a strong perpendicular magnetic field. The second goal is to study the fractional quantum Hall liquid-Wigner crystal transition by calculating accurately the energies of the Wigner crystal. In doing so, we also obtain a quantitative understanding of the sizes of various interaction effects such as exchange, intraLandau-level correlation, and inter-Landau-level correlation. We make contact with some recent experiments by comparing the present WC energy to the quantum Hall liquid energy [6]. By using different wavefunctions in the Monte Carlo calculations, we study various aspects of the physical properties of the electron solid. We find that exchange, correlation, and magnetic field effects all give rise to some interesting physical phenomena. Some of our results have been reported in a short paper [6].

In addition to the intrinsic theoretical interest of the properties of a Wigner crystal, our work has been directly motivated by the recent experimental activities looking for signatures of the electron solid in the integer quantum Hall effect (IQHE) and the fractional quantum Hall effect (FQHE) regime [10 23]. Exchange-correlation effects in these 2D electron systems in a strong magnetic field are different in essential ways from those in zero field. Most importantly, correlation-induced fluctuations are allowed, at fractional filling factors, to occur within the same Landau-level at no cost to the kinetic energy. These nearly ideal 2D systems exhibit a very rich variety of quantum phases and phase transitions [12,24,25]. 
Furthermore, the phases and the phase transitions can be controlled and studied experimentally, by changing the carrier density, the carrier effective mass, the field strength, and in some cases, the number of $2 \mathrm{D}$ layers involved. At present, we are only beginning to assess the quantitative aspects of this interesting phase diagram [6 9, 25].

In the rest of this Introduction, we make some general remarks on the problem of Wigner crystallization, discuss some recent experimental work that stimulated our investigation, and summarize our main results.

\section{A. General Remarks}

It has long been expected theoretically that at $T=0$, an interacting electron system in a uniform positive background (a jellium model), will undergo a transformation from a liquid to a solid phase as its density is lowered [1]. The Hamiltonian of the model jellium system is simply (in atomic units and in the absence of external fields):

$$
H=\sum_{i} \frac{-\nabla_{i}^{2}}{2}+\sum_{i \neq j} \frac{1}{2 r_{i j}},
$$

where interaction with a neutralizaing background is implied. The idea of the Wigner crystallization is quite intuitive. The system is characterized by the density parameter $r_{s}$ (defined in 2D by its density $n$ measured in atomic units through $\pi r_{s}^{2}=1 / n$ ). Roughly speaking, the kinetic energy of the system scales as $1 / r_{s}^{2}$ and the Coulomb interaction energy scales as $1 / r_{s}$. In a normal metal, $r_{s}$ is on the order of 1 and the kinetic energy is more important. The system is therefore characterized by the Fermi liquid theory with a well defined Fermi surface. However, if one were able to make $r_{s}$ arbitrarily large, there ought to be a crossover to a regime where the interaction becomes dominant. The resultant state is then one in which electrons are localized in a close-packed-lattice so that the average distance between them is maximized. In the total absence of the kinetic energy, the ground-state configuration of the electrons will correspond to the global minimum of the interaction potential. Properties of such an electron solid in general will not be obtainable from perturbative considerations around the liquid state: before and after the solidification, both the collective 
excitations and the single-particle excitations are qualitatively different. For example, in the solid phase, there will be a gap of the order of $e^{2} / r_{s}$ to single-particle excitations, and there will be resistance to shear. Neither occurs in the liquid phase.

Despite its theoretical certainty, direct experimental observation of the Wigner crystallization has been difficult. Only partial realization of Wigner's proposal has been achieved in a system of two-dimensional electrons trapped on the surface of liquid He [26]. In this experiment, the areal densities of the $2 \mathrm{D}$ electrons, ranging from $10^{5}$ to $10^{9} \mathrm{~cm}^{-2}$, are so low that the Fermi energy is always more than an order of magnitude smaller than the temperature at which these experiments are carried out. Therefore the system is essentially a classical one-component plasma. Nonetheless, when the interaction energy dominates over the kinetic energy, which is simply $\sim k T$ in this classical regime, one could still observe the Wigner crystallization [26]. In a three-dimensional system ( $n$-doped $\mathrm{HgCdTe}$ ), by measuring the magneto-resistance and the Hall resistance, it has been suggested that magnetically induced three-dimensional Wigner crystallization may have been realized [27].

By far the most intense experimental work in the pursuit of Wigner crystals has been carried out in quantum Hall devices [10,[1]. This regime is also more interesting since the competing liquid phase is a strongly correlated quantum Hall liquid which exhibits unusual transport properties [10,28]. Modulation doped GaAs/AlGaAs heterojunctions and silicon inversion layer devices provide an almost ideal experimental realization of a two-dimensional jellium system. Compared with electrons on helium, the lower carrier effective mass, higher density, and lower temperatures place the system in the quantum regime. Mobilities of GaAs/AlGaAs samples can be as high as $10^{7} \mathrm{~cm}^{2} /(\mathrm{Vs})$, corresponding to an effective meanfree-path almost of macroscopic length $(\sim 0.05 \mathrm{~mm})$. For hole-doped samples, mobilities are somewhat lower, $\sim 10^{5}-10^{6} \mathrm{~cm}^{2} /(\mathrm{Vs})$ [22]. In silicon MOSFETs, mobilities are still lower, around $10^{4}-10^{5} \mathrm{~cm}^{2} /(\mathrm{Vs})$, making them less ideal [23]. It has long been suggested that the application of a strong perpendicular magnetic field, which quenches the kinetic energy and confines the electrons to the size of the magnetic length $l_{B}=\sqrt{\hbar c / e B}$, will facilitate the electron crystallization [29]. Search along this direction culminated in the observation of 
an unexpected collective liquid state, i.e., the fractional quantum Hall liquid, characterized by vanishing longitudinal resistance and fractionally quantized Hall resistance [10,28].

The phase diagram of a two-dimensional electron system in a strong magnetic field is made much more intricate by the presence of such quantum Hall states. It is however still expected that ultimately Wigner crystallization would occur in a strong enough $B$-field, or a small enough Landau-level filling factor $\nu=\frac{2 \pi \hbar c n}{e B}$. In the last several years, many claims have been made, suggesting the possible observation of the Wigner crystal in both the modulation doped $G a A s / A l G a A s$ and the silicon inversion layer systems [13 19,21,23]. We defer a more detailed discussion of the current experimental situation to the next subsection.

Theoretical estimates of the crystallization density of a jellium system free of external magnetic fields have varied widely in the past [30]. The most reliable results are those given by the Green's function Monte Carlo method [3,5]. The Monte Carlo studies have for the most part focused on the body-centered-cubic lattice in $3 \mathrm{D}$ and the hexagonal lattice in $2 \mathrm{D}$ [3. $3,31,32$.

In cases where a strong magnetic field is involved, the competing liquid phase is the FQHE. Quantitative estimates for the WC transition filling factor in the quantum Hall regime can be obtained by comparing the energies of the Wigner crystal to those of the quantum Hall liquid. Various approaches have been used for this purpose. On the liquid side, exact diagonalization of small clusters with typically less than ten particles has provided much insight into the nature of the incompressible FQHE states [33]. But extrapolations to the thermodynamic limit for quantities like the ground-state energy have so far proven inaccessible with this approach. Therefore the most reliable energies for the FQHE states are obtained variationally with Laughlin's trial wavefunction which is considered very accurate [34]. Price, Platzman, and He have recently reported variational calculations of the FQHE liquid energies with a Landau-level-mixing Jastrow factor on a sphere [7]. Variational Monte Carlo calculations with planar (modified) periodic boundary condition geometry have also been carried out [8]. Both calculations give identical energies when the same trial wavefunctions are used. 
On the Wigner crystal side, strictly variational approaches have been largely limited to the Hartree-Fock approximation which ignores the crucial correlation effects [35,36. Perturbative phonon treatments beyond the harmonic level for the electron solid have been reported, but it remains unclear how fast the higher-order phonon contributions converge in the regime of density and magnetic field of experimental interest [37]. One exception that conbines the virtues of the two approaches [38 is the work by Lam and Girvin, where they optimized the variational parameters in the trial wavefunction with a truncated harmonic Hamiltonian, and then evaluated the expectation value of the original Hamiltonian with such a trial wavefunction. It captures most of the intra-Landau-level correlation, but does not treat the exchange effects, or the inter-Landau-level correlations. In addition, it has been noted that all the odd-terms in the expansion of the Hamiltonian in the phonon coordinates are not included in the total energy due to the form of the harmonic trial wavefunction [39]. The size of the third order term has been estimated [37,39].

If one compares the Wigner solid energy from Lam and Girvin in Ref. [38] with that of the FQHE liquid by Levesque, et al., in Ref. [34], the solid is favored for $\nu \leq 1 / 6.5$. However, the energy of the liquid at an arbitrary filling factor will be higher than that from interpolating between the odd-denominator filling factors [40]. This gives rise to the possibility of a reentrant WC-FQHE-WC transition as $\nu$ changes from $\nu>1 / 5$ to $\nu=1 / 5$ to $\nu<1 / 5$ as the magnetic field increases. Such reentrant phase transitions around $\nu=1 / 5$ have indeed been observed by a variety of techniques and groups, and have been mostly attributed to this mechanism [13, $19,21,23]$.

As we will discuss in the next subsection, some recent experiments have now taken us to a regime where Landau-level-mixing can not be realistically ignored [22]. In our work, we have treated exchange, intra-Landau-level correlation, and Landau-level-mixing all on equal footing [6]. It is hoped that through our work one may develop a quantitative feeling for the relative size of these effects under various experimental conditions. 


\section{B. Summary of the recent experiments}

Here, we give a brief overview of some recent experimental activities that are designed to detect the WC in the FQHE regime [13 19,21, 23]. Since the experiments are still evolving rapidly, our summary is necessarily incomplete and we apologize for any unintentional omissions.

With the very first observation of the fractional quantum Hall effect at $\nu=1 / 3$, experimentalists also encountered an insulating phase that set in at a smaller filling factor [10]. As sample quality improves, this "critical" filling factor has been pushed toward smaller values continuously. It is therefore clear that these early-observed insulating phases are due to disorder-induced localization, and not due to the interaction-induced Wigner crystallization intrinsic to a disorder-free 2D electron gas.

In the last several years, however, evidence has emerged that there are at least two insulating phases around the fractional quantum Hall state $\nu=1 / 5$ [13, 15, 17, 19,21]. Furthermore, with regards to the electron doping concentration and sample quality, these reentrant insulating phases are much more robust than the earlier insulating phases; and they become more pronounced when impurity effects are made weaker and/or when interaction effects are made stronger. In the best samples currently available (as judged by the transport gap of the FQHE state at $\nu=1 / 5$ and by the sample mobility), the insulating phases at before and after $\nu=1 / 5$ still persist [21]. The one at $\nu=0.21$ even grows in strength with sample quality, as seen from the size of the insulating gap deduced from transport measurements. This has led to the conclusion that these insulating phases are not due to disorder [21. Since disorder is not strong enough to destroy the FQHE state at $\nu=1 / 5$, it is unlikely to localize all the electrons at $\nu \geq 1 / 5$. Recall that the magneto-roton gap is very small for the $\nu=1 / 5$ FQHE state, and thus it is very susceptible to disorder 41].

Various experimental techniques have been used to study the insulating phases around $\nu=1 / 5$. These include the traditional magneto-transport [13], radio-frequency absorption [15], surface acoustic wave absorption [16, nonlinear (AC and DC) transport [14,17, 18, 21, 
noise generation [14, 18], magneto-optics [19], etc. The list is not exhaustive and is still growing. While they have revealed many interesting properties of the insulating phases, they have also brought about some controversies.

The traditional magneto-transport establishes the existence of the insulating phases [13]. Unfortunately, it does not tell us directly what gives rise to the insulating behavior. The clear stability of the $2 / 9$ and $1 / 5$ FQHE states suggests that interaction is more important than disorder [13]. Radio-frequency absorption [15], which attempts to measure the dispersion of the lower-hybrid magneto-phonon, has been fit to the characteristic $q^{3 / 2}$-dispersion, but later was fit to a $q^{1 / 2}$-mode. The latter dispersion is more likely in the presence of disorder. However it has been argued that the data can still be fit with a linear-in- $q$ dispersion [15]. This uncertainty leaves doubt as to the reliability of the interpretation of the experimental results. At least, the range of $q$ that was covered by the experiment is not wide enough to establish unequivocally the dispersion. The surface acoustic wave absorption data [16], while giving the important frequency dependence of the collective mode, make clear that in the $q$-range studied, the collective modes are very broad and unable to relate them rigorously to the existence of a Wigner crystal.

Nonlinear transport measurements have provided yet another way to study the solid 14, 17, 18,21]. A collective sliding motion would be a signature of a Wigner crystal with reasonable coherence, similar to a charge-density wave system [42]. But at present results from various groups differ in important details. For example, the sliding threshold measured in two experiments using samples with similar mobility differed by a factor of $\sim 500$. The differential conductivity above the sliding threshold became field-independent in some, but remained field dependent in others. A recent theoretical work has sought to unify some of the experimental results where a detailed comparison and analysis of the nonlinear transport experiments can be found 43]. The possible $A C-D C$ interference effects in the sliding state have also been explored both experimentally [18] and theoretically 443. Clear Shapiro steps or an inductive anomaly at a well-defined AC frequency would constitute strong evidence for the crystalline order in the insulating phases 43 . 
More direct spectroscopic tools have also been brought to bear on the FQHE/WC problem. Magneto-optical measurements have revealed several interesting aspects of the system [19,20]. First of all, spectroscopic features, rather similar to those at $\nu=1 / 3,2 / 5$, were observed at $\nu=1 / 7$ and $1 / 9$ [19]. The FQHE states at these two filling factors have so far not been definitively established in the more traditional DC transport measurements that can probe the system on a longer length scale and a lower temperature/energy scale. A possible explanation of this discrepancy was proposed based on the temperature-driven phase transitions between the WC and the FQHE [25]. There have so far been no reports of any FQHE-like feature at $\nu=1 / 11$ with the magneto-optical technique. Secondly, a second luminescence line has been observed which only appears below certain temperatures and filling factors [19]. It has been associated with the formation of a solid phase. More recent time-resolved luminescence studies seem to confirm this interpretation. Reports have been made that even the local hexagonal order in the insulating phase can be established [19. This finding is not too surprising, and while interesting, does not address the issue of long-range order in the system. Recently, it has become possible to directly observe the collective excitations of the two-dimensional electron gas in a strong magnetic field using the inelastic light scattering method [44]. Possible extension of this technique to studies of the magneto-phonon dispersion in the WC would be very interesting.

All of the earlier experiments used $n$-doped samples. However, some recent experimental work used $p$-type doping in the GaAs/AlGaAS samples [22]. The change in the carrier effective mass brings a new dimension to the problem [6,7]. Electron solidification ought to be favored by a heavier mass at comparable doping densities. It is indeed observed that the insulating phases set in in these $p$-type samples around $\nu=1 / 3$ [22], compared to $\nu=1 / 5$ in $n$-doped samples. The reentrance behavior of the insulating phases is otherwise very similar to that around $\nu=1 / 5$ in $n$-doped samples. A stringent consistency check, although not a rigorous proof, of the claims that these insulating phases are Wigner crystals, is to calculate theoretically the solidification filling factor for the $n$ - and $p$-doped samples, and to compare with what is observed experimentally. For this purpose, Landau-level-mixing must be taken 
into account since the experimental parameters are in a regime where $e^{2} / \varepsilon l_{B}$ is a factor of 10 larger than $\hbar \omega_{c}$, and since it is precisely the different amount of Landau-level-mixing that gives rise to the different transition filling factors.

Overall, there has so far accumulated a large body of suggestive evidence of the existence of this quantum solid. Most of the existing experimental data are consistent with, but none have definitively established, the formation of a Wigner crystal in these systems. It is clear that a better theoretical understanding of the properties of the electron solid under these various experimental probes will be helpful in interpreting in a more unified and consistent way the existing experimental results.

We also note that an alternative explanation for the insulating phases at $\mathrm{GaAs} / \mathrm{AlGaAs}$ heterojunctions discussed here was given by Kivelson, Lee, and Zhang, emphasizing the role of disorder [24]. A generic phase diagram was constructed in terms of the Landaulevel filling factor and the strength of disorder [24]. While appealing and possibly relevant to experiments done on samples with much more disorder, there are difficulties with this theory when applied to experiments carried out on the best quality samples. As we already mentioned, the insulating phases become stronger as disorder potential is weakened in these samples, which is difficult to understand within the framework of this theory. There are also some recent experimental results which are in apparent contradiction with the predicted phase diagram 13,22].

\section{Summary of our main results}

Our work can be separated into two parts: $B=0$ and $B \neq 0$. We summarize them in what follows.

In the case of no external magnetic field, we have studied the variational energies of electrons localized on various lattices, including the square lattice, the honeycomb lattice, and the hexagonal lattice [32]. In investigating the lattice dependence of the ground-state spin-ordering, we have also studied the rectangular lattice. The hexagonal lattice is found to 
have the lowest energy, in agreement with classical Ewald energy considerations. We also find that the square lattice favors slightly the ferromagnetic (FM) state whereas the honeycomb lattice favors the antiferromagnetic (AFM) state. This is despite the fact that both are bi-partite lattices. The energies of different spin states on a hexagonal lattice are very close, and beyond the resolution of the present variational Monte Carlo calculations. The physics of the electron solid appears very rich even in the absence of an external magnetic field. We propose that the many-electron ring-exchange mechanism is responsible for the calculated behavior 45 47]. We will also discuss the ring-exchange processes in the presence of a strong magnetic field, which have been invoked as an alternative picture for the quantum Hall liquid 48 51.

In the IQHE and the FQHE regime, we find that exchange effects are unimportant in the regime of density and magnetic field near the Wigner crystallization point in these systems. In $n$-doped samples, intra-Landau-level correlations are the most important, and are well represented by the magneto-phonon wavefunction proposed by Lam and Girvin [38. For p-doped samples, however, Landau-level-mixing is the single most important mechanism for lowering the energy of the WC, in comparison with exchange and intra-Landau-level

correlation. However even when $e^{2} / \varepsilon l_{B}$ is 10 times $\hbar \omega_{c}$, intra-Landau-level correlations still make a very large contribution to the total energy. Combining our WC energies with those of the FQHE liquid with Landau-level-mixing, we find that the solidification in $p$-doped samples will occur around $\nu=1 / 3$ [6, []. This is in nice agreement with the recent experiments performed on $p$-doped samples [22]. Effects of finite temperatures and of disorder, and Wigner crystallization at integer filling factors will also be discussed.

\section{Outline of the paper}

The balance of the paper is as follows. In Sec. II, we focus on the spin-ordering in the ground-state of Wigner crystal in various 2D lattices with no magnetic field. We interpret the results with a picture of multi-electron exchange interaction. We then comment on the 
ring-exchange processes in the presence of a strong magnetic field, and examine some overall characters of our trial wavefunctions. We begin our investigation in the FQHE regime in Sec. III where we give the details of the present VMC calculation in a strong magnetic field. Our choice for the form of the variational wavefunction is motivated. Results, comparison to previous work, and possible implications for the current experiments are given in Sec. IV. We conclude in Sec. V.

Those who are only interested in cases with a strong magnetic field may go to Secs. IIC, III and IV directly.

\section{2D WIGNER CRYSTALS IN THE ABSENCE OF A MAGNETIC FIELD: SPIN-ORDERING}

In this section, we focus on the spin-ordering of electrons interacting with the long-range $1 / r$ Coulomb potential on various two-dimensional lattices with the variational quantum Monte Carlo method. Some comments are made for the ring-exchange processes involving a strong magnetic field. A general analysis of our trial wavefunction and its possible generalization to other cases are also given.

\section{A. Results from VMC: possible role of many-electron exchange}

The spin-ordering of electrons on several two-dimensional lattices is found to depend strongly on the underlying lattice. We have considered the square (SQ) lattice, the honeycomb (HC) lattice and the hexagonal (HX) lattice, in the absence of any external magnetic field. We focus on the first two crystal structures because they appear to behave very differently despite the fact that both are bipartite. Within our variational wavefunctions, the FM state is favored on the square lattice by a much smaller margin than one by which the AFM state is favored on the honeycomb lattice. In addition, the rectangular lattice is studied as a model that can continuously vary from a square lattice to a collection of interacting

chains in 2D. We find a transition from an FM ground-state to an AFM ground-state as the 
aspect ratio deviates from one. These results demonstrate the importance of many-particle exchange effects for fermions interacting with a $1 / r$ potential in $2 \mathrm{D}$. They also show that the relative importance of the exchange processes involving different number of electrons depends strongly on the lattice geometry.

The notion of many-particle exchanges was previously invoked 45,46] in attempts to understand the magnetic properties of three-dimensional solid ${ }^{3} \mathrm{He}$. It is now well-known $45,46,52,53$, 53 that these many-particle exchanges dominate the magnetic properties of solid ${ }^{3} \mathrm{He}$ in both $2 \mathrm{D}$ and $3 \mathrm{D}$. That these ring-exchanges may also affect the magnetic properties of the electron Wigner crystal was suggested by Herring 477 in the 1960's. Variational and Green's function Monte Carlo calculations were carried out previously, but only for the 2D hexagonal lattice [5]. Two spin-orderings were considered. One is the FM state and the other one has electrons of opposite spins aligned on alternating chains in the hexagonal lattice. The latter is not a true AFM state since the hexagonal lattice is not bipartite. They were found to have the same energy within the statistical noise in the calculation. The ground-state of the spin- $\frac{1}{2}$ nearest-neighbor Heisenberg antiferromagnet on a hexagonal lattice has been a subject of tremendous amount of work [54]. To clarify the spin-ordering of a 2D hexagonal $\mathrm{WC}$ is a much more demanding task still. A semi-classical $W K B$ estimate of the various ring-exchange frequencies suggests that the three-particle exchange may be more important than both the two- and four-particle exchanges 46.

The trial wavefunction used in the present VMC calculations is of the Jastrow-Slatertype:

$$
\psi=\exp \left[-\frac{1}{2} \sum_{i \neq j} u\left(\vec{r}_{i}-\vec{r}_{j}\right)\right] D_{\uparrow} D_{\downarrow} .
$$

Here $D_{\uparrow}$ and $D_{\downarrow}$ are respectively the Slater determinants for spin-up and spin-down electrons on the lattice. The single-particle orbitals $\phi(\vec{r})$ in the Slater determinants are taken to be in most cases isotropic Gaussians localized about the lattice sites $\vec{R}_{i}, \phi_{i}=e^{-\left(\vec{r}-\vec{R}_{i}\right)^{2} / r_{G}^{2}}$, except for the rectangular lattice (see below). The width $r_{G}$ is a variational parameter to be optimized. Additional variational degrees of freedom in the one-particle orbitals are 
introduced in further investigating the AFM state on the square lattice. The two-particle correlation factor in Eq. 2 is taken to be of the form:

$$
u(r)=\frac{A}{\sqrt{r}}\left(1-e^{-\sqrt{r / F}-\frac{1}{2} r / F}\right) .
$$

It has a long range tail of $\frac{A}{\sqrt{r}}$. This, in the absence of the Gaussian single-particle orbitals in the Slater determinants, yields a longitudinal phonon dispersion $\propto q^{1 / 2}$ for small- $q$ which is a result of the Coulomb interaction in 2D. In the limit of $r \rightarrow 0, \frac{d u}{d r}=-\frac{A}{3}\left(\frac{1}{F}\right)^{3 / 2}$ and $u=A / \sqrt{F}$. With properly chosen $\mathrm{A}$ and $\mathrm{F}$, the short range cusp-condition [55] can be satisfied. We have determined these two parameters in our trial wavefunction variationally. The optimal values are always very close to those given by the cusp-conditions and the long wavelength longitudinal phonon considerations. Calculations are done using the Metropolis algorithm with periodic boundary conditions in 2D.

Our results for the hexagonal structure are the same as those of existing VMC calculations reported previously where a somewhat different Jastrow factor was used [5]. In Table I, we show a comparison between the present VMC calculation and a previous VMC calculation [5] for a simulation cell containing 56 electrons. Finite size effects have been examined using different size simulation cells. To the significant digits given in Table I, they are negligible. For reference, results from a fixed-node Green's function Monte Carlo calculation [5] are also shown. As mentioned above, because of the frustration effects, the energy differences between different spin-orderings of the $\mathrm{WC}$ on the hexagonal lattice are too small to be studied with the present method. From now on, in discussing the spin structures, we will restrict ourselves to the SQ lattice and the $\mathrm{HC}$ lattice.

The energies here are dominated by the classical Ewald energy. For the three lattices studied here, they are respectively (in atomic units): $E_{\text {Ewald }}=-1.10610 / r_{s}$ for the HX lattice, $E_{\text {Ewald }}=-1.10024 / r_{s}$ for the SQ lattice, and $E_{\text {Ewald }}=-1.06841 / r_{s}$ for the HC lattice. At a given density, the $\mathrm{HC}$ lattice has the smallest nearest-neighbor distance since it is the least close-packed among the three, hence it has the highest classical energy. We find that quantum effects tend to reduce the energy differences, but they are not large enough 
to reverse the ordering. In Table II, we show, for these three lattices, the VMC energies calculated at $r_{s}=30,50,70$, and 100, all with complete spin polarization. Again, finite size effects are negligible compared to the significant digits given.

We now focus on the different spin-orderings in the SQ and HC lattices. In the present work, the FM state is formed with all the single-particle orbitals having the same spin. Thus the total wavefunction contains only one Slater determinant. The AFM state on these bipartite lattices is constructed by occupying the single-particle orbitals on one sublattice with spin-up electrons and the other with spin-down electrons. The spin-dependent cuspconditions 55 are satisfied with a spin-dependent Jastrow factor. In Figs. 1 and 2, we show the energy differences between the FM state and the AFM state on the SQ lattice and the $\mathrm{HC}$ lattice for several $r_{s}$. As we can see, the FM state is slightly lower in energy than the AFM on the square lattice. But on the honeycomb lattice the AFM is lower in energy by a substantial margin for $r_{s} \leq 100$. Table III illustrates that the finite size effects at $N \sim 50$ are already negligible. Results reported in the figures are calculated with the largest simulation cells shown in Table III.

In general, it is more difficult to construct a good trial wavefunction for a more disordered state. In the present case, one might suspect that the FM state is slightly favored over the AFM state in the variational calculations. Of course, this will not change our conclusion regarding the $\mathrm{HC}$ lattice, where the more disordered phase (AFM state) already has a lower energy. For the SQ lattice, we have made the following two attempts to lower the energy of the AFM state:

1). Instead of having Gaussians localized on a single lattice site in the one-particle orbitals, we have used:

$$
\phi_{j}(\vec{r})=e^{-\left(\vec{r}-\vec{R}_{j}\right)^{2} / r_{G}^{2}}+C \sum_{i=n n} e^{-\left(\vec{r}-\vec{R}_{i}\right)^{2} / r_{G}^{2}}
$$

with proper normalization factors. Here $C$ is a variational parameter and $\sum_{i=n n}$ indicates summing over the nearest neighbor sites. This form of $\phi(\vec{r})$ distributes some weight of the one-particle orbital onto its neighboring sites. Noting that the nearest neighbors have the 
opposite spin, we expect that the sublattice magnetization of the $2 \mathrm{D}$ antiferromagnet could be adjusted by changing $C$.

2). We have also tried one-particle orbitals similar to the above, but with some weight on the hollow sites in the lattice rather than on the neighboring lattice sites. The motivation was to lower the kinetic energy at minimal cost to the interaction energy.

In both of these cases, the AFM state energy on the SQ lattice was not lowered to within our statistical accuracy. Despite these efforts, we can not be absolutely certain that the FM state is lower in energy than the AFM state on the SQ lattice. In fact, we do not know if the ground-state of a WC on a SQ lattice would be magnetically ordered at all. The closeness of these two states on a SQ lattice leaves open the possibility that the ground-state on a square lattice may have a more subtle magnetic ordering than what we are able to examine in the present variational calculations. It is safe to conclude, however, that our calculations illustrate clearly the qualitative difference between the bipartite square and honeycomb lattices in their ground-state spin-ordering.

\section{B. Discussions and further tests}

For fermions on a lattice, it has been argued by Herring 477 and by Thouless 45 that, in general, ferromagnetism is favored by ring-exchange processes involving odd number of particles $((2 n+1)$-exchange), whereas antiferromagnetism is favored by ring-exchange processes involving even number of particles (2n-exchange). A heuristic argument may go as follows. Take the example of an arbitrary $(2 n+1)$-ring-exchange. A ring-exchange

of $(2 n+1)$-particles is an even permutation. Thus the total wavefunction must keep the same sign upon this ring-exchange. To minimize the energy, one would like to keep the number of nodes in the spatial part of the wavefunction minimal. Thus, a wavefunction in which the spatial part is totally symmetric will be favored. We then must make the spin part of the wavefunction totally symmetric as well. Consequently, the FM state, where all spins are aligned, will be favored by such exchanges. Similarly one concludes that AFM 
is favored by $2 n$-exchanges. Following this line of reasoning, our results suggest that twoparticle exchanges are more important on the $\mathrm{HC}$ lattice while three-particle exchanges are relatively more important on the SQ lattice. The competition between them determines the ground-state spin-ordering.

Compared with the interaction potential in solid ${ }^{3} \mathrm{He}$ [46], the $1 / r$ Coulomb interaction is much softer for small $r$. This results in lesser steric constraint in exchanges involving fewer particles. One might therefore speculate that exchange frequencies as a function of $n$ will decay faster for electrons on $2 \mathrm{D}$ lattices than for solid ${ }^{3} \mathrm{He} 46$. These plausibility arguments could be checked by either the semi-classical $W K B$ approach [46] or by using the path-integral Monte Carlo approach [52] which has been applied to study the ring-exchange frequencies in body-centered-cubic solid ${ }^{3} \mathrm{He}$.

Although the variational Monte Carlo method was the first [53] to be used in studying numerically the four-particle exchange frequency in solid ${ }^{3} \mathrm{He}$, it has later been shown to be quantitatively unreliable [46,52]. This is largely due to the fact that in the case of solid ${ }^{3} \mathrm{He}$ the exchange energies are only $10^{-4}$ th of the typical phonon energies. Thus an excellent variational wavefunction for the total energy might be inadequate for estimating the exchange frequencies. In the present case however, the energy differences between the $\mathrm{AFM}$ and the FM spin-orderings at $r_{s}=30$ are about $1 \%$ for $\mathrm{SQ}$ and $5 \%$ for $\mathrm{HC}$ of the total zero-point-motion energy. In fact for $\mathrm{HC}$ lattice, $E_{F M}-E_{A F M}$ at this $r_{s}$ approaches the total energy difference between the SQ lattice and the HC lattice. We therefore believe that the qualitative difference in the ground-state spin-ordering between these two bipartite lattices is not an artifact of the variational method.

The energy splitting between the FM state and the AFM state is found to be very sensitive to the Gaussian width $r_{G}$ in the one-particle orbital. In Fig. 3, we show this sensitivity for the HC lattice. While the sharp drop on the small $r_{G}$ side may be attributed to the exponential decrease of the exchange overlap integral, the somewhat slower drop on the large $r_{G}$ side could be due to the different $r_{G}$-dependences of the two-particle and threeparticle ring-exchange processes. The energy splitting peaks at an $r_{G}$ smaller than one which 
optimizes the energies.

Further support for the importance of the multi-particle ring-exchanges for electrons on a $2 \mathrm{D}$ lattice is given by studying the rectangular lattice as it varies from a square lattice to a set of weakly interacting chains. Here, we have used anisotropic Gaussians in accordance with the aspect ratios. The sizes of the Gaussians along different directions are kept proportional to the two side lengths of the rectangle. In the essentially one-dimensional limit of a set of weakly interacting chains, two-particle exchange is expected to dominate. This is because multi-particle exchanges will involve tunneling over longer distances with the same or higher potential barriers than the two-particle exchange in this limiting case. Consequently the AFM state should have a lower energy if the aspect ratio is large enough. In Fig. 4, we show the energy difference between the FM state and the AFM state on a rectangular lattice as a function of its aspect ratio for $r_{s}=30$. Indeed, we see a transition in the region of $a / b=1.15$ to $a / b=1.20$. All of these observations from our calculations are consistent with the multi-electron ring-exchange picture. We thus believe that the ring-exchange processes play an important role in determining the ground-state spin structure for electrons on these 2D lattices.

\section{Many-particle exchanges in the Wigner crystal in a strong magnetic field}

It is of interest to ask how the presence of a strong magnetic field may affect the manyparticle exchange processes. In two-dimensions, at the mean-field-theory level, at suitable magnetic filling factors, the physical magnetic field may be gauged away [24]. One is then left with composite particles which carry flux quanta moving in an effective zero magnetic

field. Since statistics of the particles may be altered in such formal transformations of the Hamiltonian, and since the exchange interaction is intimately related to the statistics, it is expected that the many-particle exchange effects may be dramatically affected by a magnetic field.

This issue has been taken up in a series of papers by several authors in which they 
argued at filling factors such as $\nu=1 / 3,2 n$ and $(2 n+1)$-exchanges will add coherently and yield a condensation of the large ring-exchange processes 48,49. This notion as it was first proposed [48], while appealing physically, cannot survive the large magnetic field limit on a lattice. In the resultant localized crystal phase, the exchange interactions can be made arbitrarily small. Our numerical calculations also show clearly that exchange processes are unimportant compared to the correlation effects in a magnetically induced Wigner crystal. Within our numerical resolution, the total energy changes smoothly with the applied magnetic field, or with the magnetic filling factor $\nu$, showing no downward cusps necessary for the experimentally observed incompressibility at odd-denominator filling factors.

This problem of invoking the lattice is circumvented later by Baskaran and Lee, Baskaran, and Kivelson 49] who showed that the crystal phase is not necessary for such a condensation to occur, although it is convenient for the sake of visualization. There is also a formal mapping between the density matrix from the Laughlin wavefunction and that from the condensed ring-exchange processes 49].

Thouless and Li have raised another criticism, regarding the sign of the ring-exchanges at filling factors that are the inverse of odd integers [50]. We repeat their argument for spin-polarized systems with the following diagram (Fig. 5). The uparrows (the downarrows) indicate that the energy goes up (down) when such exchanges are included. One might then conclude that the ring-exchange processes at odd-denominator filling factors would in fact make them energetically less favored compared to its immediate neighboring filling factors. Therefore, the energy will show an upward cusp at odd-denominator filling factors, instead of the downward cusp as implied by the observation of the FQHE [10,28].

We think that this argument is in fact not valid in the FQHE regime [51] because it rests on the assumption that the energy always goes up as the number of nodes in the spatial part of the wavefunction increases. We argue below that existing calculations explicitly show that this is not the case for the very system of interest here.

We make use of a recent work by Xie, He, and Das Sarma where they considered two 
systems of identical interacting particles on a sphere [56]. The only difference between the two is that one is bosonic and the other fermionic. In the absence of a magnetic field, the Bose system will in general have a lower energy [57]. But in the presence of magnetic field, the urge to lower the interaction energy and the possibility of doing so without costing kinetic energy make it under certain circumstances favorable to have nodes in the manybody wavefunction. This is indeed what was found from the exact diagonalization of the few particle system [56: At filling factors $\nu=1 / 2$, the bosonic system has a lower energy but at $\nu=1 / 3$ the fermionic system has a lower energy for Coulomb interactions. The qualitative trend appears to continue for all the filling factors examined [56]. As the magnetic field decreases or as the filling factor increases to well above 1, eventually the Bose system will have a lower energy.

\section{Qualitative features of the trial wavefunction}

In order to gain a more physical understanding about what kind of correlations are included in our trial wavefunction, it is instructive to transform our wavefunction in terms of the phonon coordinates. This transformation cannot be carried out in the most general case, similar to earlier work on the liquid phase [58]. We therefore ignore exchange, i.e., approximate the Slater determinants $D_{\uparrow} D_{\downarrow}$ with:

$$
D_{\uparrow} D_{\downarrow}=e^{-\sum_{i=1}^{N}\left(\vec{r}_{i}-\vec{R}_{i}\right)^{2} / r_{G}^{2}} .
$$

Using:

$$
\frac{1}{2} \sum_{i \neq j} u\left(r_{i j}\right)=\frac{1}{2 N} \sum_{\vec{q}} u(q)\left[\rho_{\vec{q}} \rho_{-\vec{q}}-N\right],
$$

where

$$
\rho_{\vec{q}}=\sum_{j=1}^{N} e^{i \vec{q} \cdot \vec{r}_{j}}
$$

we can rewrite our original wavefunction, Eq. 2, as: 


$$
\begin{aligned}
\psi_{T}\left(\vec{r}_{1}, \vec{r}_{2}, \ldots, \vec{r}_{N}\right) & =e^{-\frac{1}{2} \sum_{\vec{q}} u(q)\left[\rho_{\vec{q}} \rho_{-\vec{q}}-N\right] / N-\sum_{i=1}^{N}\left(\vec{r}_{i}-\vec{R}_{i}\right)^{2} / r_{G}^{2}} \\
& =e^{K}
\end{aligned}
$$

which defines $K$.

Under the assumption that the deviations of $\vec{r}_{i}$ from $\vec{R}_{i}$ are small, we may expand $\rho_{\vec{q}}$ to first order in $\vec{\xi}_{i}=\vec{r}_{i}-\vec{R}_{i}$, and obtain for $K$ :

$$
K=-\frac{1}{2} \sum_{\vec{k}} \vec{\xi}_{\vec{k}} \cdot\left(\vec{k} u(k) \vec{k}+2 \mathbf{I} / r_{G}^{2}\right) \cdot \vec{\xi}_{-\vec{k}}
$$

where $\mathbf{I}$ is the unit matrix and the phonon coordinates $\vec{\xi}_{\vec{q}}$ are defined as:

$$
\vec{\xi}_{\vec{q}}=\frac{1}{\sqrt{N}} \sum_{i=1}^{N} \vec{\xi}_{i} e^{-i \vec{q} \cdot \vec{R}_{i}}
$$

This form is simply a product of $N$-noninteracting simple harmonic oscillators. The kernal $\vec{k} u(k) \vec{k}+2 \mathbf{I} / r_{G}^{2}$ can be diagonalized, yielding:

$$
\omega_{L}=2 / r_{G}^{2}+u(k) k^{2}
$$

and

$$
\omega_{T}=2 / r_{G}^{2}
$$

where $\omega_{L}$ is the eigenfrequency of the longitudinal mode from the diagonalization of the tensor, and $\omega_{T}$ is the transverse mode frequency. In $D$-dimensions, there are $D-1$ degenerate transverse modes from this simple analysis.

The physics of this line of reasoning is quite clear. The isotropic Gaussians are by themselves $N$ uncoupled $D$-dimensional isotropic harmonic ocsillators, giving a finite frequency to both the transverse and the longitudinal modes. The correlation factor $u(r)$ in its present form is only a function of inter-particle distance, thus only affects the density fluctuations in the long-wavelength limit where our phonon expansion is valid. This is precisely why $u(r)$ only enters the frequency of the longitudinal mode which couples to density fluctuations.

It is known, for Coulomb interacting systems, long wavelength density fluctations have a finite frequency in $3 \mathrm{D}$ while in $2 \mathrm{D}$ it disperses as $\sqrt{q}$. These statements regarding the 
longitudinal fluctuations are true for both the solid and the liquid phases. They may be used to determine the asymptotic behavior of the optimal $u(r)$ used in the variational calculations. We note that the $\sqrt{q}$-dispersion for the longitudinal phonon in $2 \mathrm{D}$ is violated in the long-wavelength limit by our variational wavefunction. Similarly, the linear-in- $q$ dispersion of the transverse phonon is also violated. This observation suggests that, while our wavefunctions may be very accurate for ground-state energies, it can not be used directly for calculating phonon frequencies in the small $q$-limit. We remark that similar violations of the long-wavelength phonon dispersions in electron Wigner crystals with no external magnetic fields, and in helium solids also appear in nearly all of the previous variational Monte Carlo calculations.

Not only does this transformation provide some insight into our trail wavefunction, it can also be used constructively to find an optimal $u(k)$ in certain cases. In the liquid phase, the approximation equivalent to the one we made above for the solid is the random-phaseapproximation for the total energy, which is then minimized with respect to $u(\vec{k})$ [58. As an illustration of the general principle, we have also applied this idea to the problem of bcc solid hydrogen in the Mott insulating regime and derived an optimal electron-electron correlation factor. In this case, we find:

$$
2 u(k)=-1+\sqrt{1+\frac{4 m}{\hbar^{2} k^{2}}\left(v_{k}+2 \vec{k} \cdot \mathbf{s} \cdot \vec{k} / k^{4}\right)},
$$

where

$$
\mathbf{s}=-\frac{e^{2}}{2} \sum_{j \neq 0} \frac{3\left(\vec{R}_{j}-\vec{R}_{0}\right)\left(\vec{R}_{j}-\vec{R}_{0}\right)-\mathbf{I}\left(\vec{R}_{j}-\vec{R}_{0}\right)^{2}}{\left|\vec{R}_{j}-\vec{R}_{0}\right|^{5}}
$$

and the Fourier transform of the Coulomb interaction in 3D is:

$$
v_{k}=4 \pi e^{2} / k^{2}
$$

Here $\vec{R}_{0}$ is the position of an arbitrary proton in the bcc lattice and $\vec{R}_{j}$ are the positions of all the protons. In the absence of the proton lattice structure, which amounts to simply setting $\mathbf{s}=0$ as one can verify in the course of derivation, we have 


$$
2 u(k)=-1+\sqrt{1+4 m v_{k} / \hbar^{2} k^{2}} .
$$

The problem is reduced to that of a jellium and the above result agrees with earlier work for this quantity [58].

\section{ENERGY OF A WIGNER CRYSTAL IN FQHE REGIME: METHOD}

In the rest of this paper, we focus on cases where a large magnetic field is involved. Effects of exchange, intra-Landau-level correlation, and Landau-level-mixing on the total energy, and their dependence on the carrier mass and magnetic field strength are examined on equal footing. In Sec. IIIA, we briefly review the problem. In Secs. IIIB and IIIC, we describe the Hamiltonian and the variational wavefunctions used, and the technical details in their evaluation along the Monte Carlo walks. In Sec. IIID, we assess the quality of the trial wavefunction. In this section, we focus more on the qualitative aspects of our calculations. Numerical results and discussions are presented in Sec. IV.

\section{A. Brief overview}

There have been many studies on $B$-field induced Wigner crystals in 2D. Most of these were carried out within the Hartree-Fock approximation [35,36]. An alternative approach has been to expand the Hamiltonian in terms of phonon coordinates and to seek to perturbatively

improve the results [37,39]. The former is variational but contains no information regarding the crucial correlation effects. The latter is no longer variational and existing calculations show that the rate of convergence is unsatisfactory. With a few exceptions [6, 36, 59], only the lowest Landau-level states were considered.

The present approach has the advantage of being variational. In addition, by varying the trial wavefunctions used, we are able to obtain a quantitative understanding of the roles played by exchange, intra-Landau-level correlation, and Landau-level-mixing in a Wigner crystal. Special attention is paid to the interplay between these effects and the experimental 
parameters: the carrier density, carrier mass, and the strength of the magnetic field. From our calculations, we find that the effects of Landau-level-mixing are indeed large enough to account for the observed difference in $\nu_{c}$ between the eletron and the hole GaAs/AlGaAs systems. For GaAs, the relevant materials parameters are: $\varepsilon=13$, electron effective mass $m_{e}^{*}=0.067 m_{e}$, and heavy-hole effective mass $m_{h}^{*}=0.35 m_{e}$. Experimentally for the present heterostructure, the heavy-hole effective mass is less certain 60]. For the present heterojunction system in the strong magnetic field limit, we only need to consider the heavy-hole band for $p$-doped samples. We now describe details of the present VMC calculations involving a strong magnetic field.

\section{B. Present VMC calculations: the Hamiltonian}

The exact Hamiltonian for 2D electrons of effective mass $m^{*}$ in a magnetic field is given by:

$$
H=\sum_{i} \frac{\left(\vec{p}_{i}+e \vec{A}\left(\vec{r}_{i}\right)\right)^{2}}{2 m^{*}}+\frac{e^{2}}{2 \varepsilon} \sum_{j \neq i} \frac{1}{r_{i j}} .
$$

Here $\vec{A}$ is the vector potential, $\vec{A}=(-y B / 2, x B / 2)$ in symmetric gauge with $\vec{B}$ in $+z$ direction. $\varepsilon$ is the dielectric constant of the host material $\left(\frac{e^{2}}{\varepsilon}=m^{*}=1\right.$ in effective atomic units). The Zeeman term is left out of Eq. 17 since we assume total spin-polarization.

In choosing a particular gauge for the vector potential $\vec{A}$ in the Hamiltonian, we must also choose an origin, which breaks the continuous translational invariance. As a result of the generalized periodic boundary conditions [61], all the rational fields can be studied directly in our numerical work. Properties of the WC at irrational fields can only be obtained to the extent continuity holds for the particular physical quantity. In Sec. IVF, we provide

numerical evidence that there is no cusp in the WC total energy as a function of filling factor.

A finite simulation cell with modified periodic boundary conditions [61] is used, and only the hexagonal lattice is considered in view of the results presented in Sec. II. With the 
kinetic energy quenched by the magnetic field, it is expected that the hexagonal structure would be made even more stable (compared to the $B=0$ case) than the other $2 \mathrm{D}$ lattices.

The evaluation of the $1 / r$ interaction energy at each step of the Monte Carlo walk is not affected by the magnetic field. It is done with the usual Ewald sum method in $2 \mathrm{D}$ at each step of the Monte Carlo walk. To evaluate the kinetic energy, let us first define:

$$
\begin{aligned}
& u=x+i y, \\
& v=x-i y .
\end{aligned}
$$

The kinetic energy operator is now:

$$
\begin{aligned}
K . E . & =\sum_{i} k . e .^{(i)} \\
& =\frac{1}{2 m^{*}} \sum_{i}\left(\vec{p}_{i}+e \vec{A}\left(\vec{r}_{i}\right)\right)^{2} \\
& =\frac{1}{2 m^{*}} \sum_{i}\left\{-4 \frac{\partial^{2}}{\partial u_{i} \partial v_{i}}+\frac{1}{l_{B}^{2}}\left(u_{i} \frac{\partial}{\partial u_{i}}-v_{i} \frac{\partial}{\partial v_{i}}\right)+\frac{u_{i} v_{i}}{4 l_{B}^{4}}\right\} .
\end{aligned}
$$

The local kinetic energy

$$
K \cdot E \cdot \text { loc }=\frac{1}{\psi} \sum_{i} \frac{1}{2 m^{*}}\left(\vec{p}_{i}+e \vec{A}\left(\vec{r}_{i}\right)\right)^{2} \psi,
$$

is transformed into the following form:

$$
\begin{aligned}
2 m^{*} K \cdot E_{\cdot l o c}= & -4\left(J_{u v}+D_{u v}\right)-4\left(J_{u} \cdot J_{v}+J_{u} \cdot D_{v}+D_{u} \cdot J_{v}\right) \\
& +\frac{1}{l_{B}^{2}}\left(u \cdot D_{u}-v \cdot D_{v}\right)+\frac{1}{l_{B}^{2}}\left(u \cdot J_{u}-v \cdot J_{v}\right)+\frac{u v}{4 l_{B}^{4}},
\end{aligned}
$$

where the many-body wavefunction is written as

$$
\psi\left(\vec{r}_{1}, \vec{r}_{2}, \ldots, \vec{r}_{N}\right)=J\left(\vec{r}_{1}, \vec{r}_{2}, \ldots, \vec{r}_{N}\right) \cdot D\left(\vec{r}_{1}, \vec{r}_{2}, \ldots, \vec{r}_{N}\right),
$$

and,

$$
J_{u}=\frac{\partial \ln J}{\partial u}
$$




$$
\begin{gathered}
J_{v}=\frac{\partial \ln J}{\partial v}, \\
J_{u v}=\frac{\partial^{2} \ln J}{\partial u \partial v}, \\
D_{u}=\frac{\partial \ln D}{\partial u}, \\
D_{v}=\frac{\partial \ln D}{\partial v}, \\
D_{u v}=\frac{\partial^{2} \ln D}{\partial u \partial v}+D_{u} \cdot D_{v} \\
=\frac{1}{D} \frac{\partial^{2} D}{\partial u \partial v} .
\end{gathered}
$$

The magnetic length $l_{B}$ is $\sqrt{\hbar c / e B}$ as before. We have suppressed the electron index $i$ in all the terms on the right-hand-side of Eq. 21. Each of them is collected along the walk and used to construct the local kinetic energy at a given configuration. The above transformation is not necessary — one can in fact just as easily evaluate the local kinetic energy in terms derivatives with respect to $x$ and $y$, although it does make the distinction between lowest Landau-level states and the higher Landau-level states more apparent. This is because the lowest Landau-level wavefunctions can be written as a product of a Gaussian with an analytic function of $u$. In other words, a many-body wavefunction restricted to the lowest Landau-level states will not contain any $v$-dependence in its Jastrow factor $J$, that is, $J_{v}=J_{u v}=0$.

Given a trial wavefunction, we sample the total energy with the Metropolis scheme [2]. The fairly strong localization of the electron wavefunction makes it possible for us to totally eliminate the finite size effects. (The fact that we are dealing with a 2D systems also reduces the total number of particles needed for convergent results compared with a 3D system of the same linear size.) Most of our numerical calculations are carried out for a simulation cell with 100 spin-aligned electrons. Calculations with different size simulation cells show that the resulting finite size effects are smaller than the statistical noise in our results. We 
will give more details regarding finite size convergence. No finite size scaling is needed to extrapolate the energy to the thermodynamic limit. The numerical aspects of the calculation are therefore well under control.

\section{Many-body wavefunction}

Denoting the simulation cell by vectors $\vec{L}_{x} \times \vec{L}_{y}$, we may write the single-particle orbital that is localized about the lattice site $\vec{R}_{j}$ and satisfies the generalized periodic boundary conditions as:

$$
\phi_{j}(\vec{r})=\frac{1}{\sqrt{2 \pi}} \frac{\beta}{l_{B}} \sum_{\vec{T}} \exp \left\{-\frac{\beta^{2}}{4 l_{B}^{2}}\left(\vec{r}-\vec{R}_{j}-\vec{T}\right)^{2}+\frac{i}{2 l_{B}^{2}}\left[\vec{r} \times \vec{R}_{j}+\vec{r} \times \vec{T}+\vec{R}_{j} \times \vec{T}\right]_{z}\right\}
$$

Here $\vec{T}=n_{x} \vec{L}_{x}+n_{y} \vec{L}_{y}$ with arbitrary integers $n_{x}$ and $n_{y} . \beta$ is a variational parameter which determines the localization of the wavefunction. Changing $\beta$ does not affect the phase factors appearing in $\phi(\vec{r})$. (For $\beta=1, \phi(\vec{r})$ lies entirely within the lowest Landau-level.)

One may form either a Slater determinant or a simple product of these single-particle orbitals. When multiplied by a purely periodic Jastrow factor, both of the resultant manybody wavefunctions satisfy the generalized periodic boundary condition in a vector potential [61]. Without the Jastrow factor, none contains correlation but the former does contain exchange. Therefore, we can obtain a rigorous upper bound for the size of exchange energy using these wavefunctions.

We now motivate the Jastrow factor that we use. It consists of two parts: One is the same as that in the absence of the magnetic field which is shown to be quite accurate in that case; the second part, arising from the zero-point-motion of the magneto-phonons, is peculiar to cases involving a strong magnetic field. The latter is found to be more important in terms of its effects on energy. Of course, such a separation is not entirely strict.

Our derivation for the magneto-phonon correlated wavefunction is slightly different from Lam and Girvin's work [38]. They adopted the lowest Landau-level approximation at the outset and sought to optimize the harmonic Hamiltonian within the lowest Landau-level sub- 
Hilbert space. We have chozen to solve the harmonic Hamiltonian exactly without making the lowest Landau-level approximation. We can then obtain the Lam-Girvin lowest Landaulevel magneto-phonon wavefunction by taking the large magnetic field limit. Clearly these two procedures are equivalent in the strong-field limit, although our scheme is somewhat more flexible as it may be used to include some of the Landau-level-mixing effects. Our derivation closely parallels that of Chui and his coworkers' [37, although they have focused more on the eigenvalues than on the wavefunctions.

After we take the large field limit, our wavefunction is the same as that given by Lam and Girvin 38]. Using their notation, the wavefunction $\psi_{\text {cor }}$ restricted to the lowest Landau-level is:

$$
\psi_{\text {cor }}=\exp \left[\frac{A_{p}}{4 l_{B}^{2}} \sum_{i, j} \xi_{i} B_{i j} \xi_{j}\right] \prod_{i} \phi_{i}\left(\vec{r}_{i}\right),
$$

with $A_{p}=1$. $\phi_{i}$ 's are the single-particle orbitals in Eq. 24 without the $\vec{T}$ 's. $\xi_{i}$ is the displacement of the ith-electron from the lattice site $R_{i}$, written in complex coordinates. The $B_{i j}$ 's, appearing in the Jastrow factor, couple the motion of the $i t h$-electron with that of the $j$ th-electron. Its Fourier transform $B(\vec{k})$ is:

$$
B(\vec{k})=e^{i \theta_{\vec{k}}} \frac{\omega_{L}^{0}(\vec{k})-\omega_{T}^{0}(\vec{k})}{\omega_{L}^{0}(\vec{k})+\omega_{T}^{0}(\vec{k})},
$$

with,

$$
\begin{aligned}
& \cos \theta_{\vec{k}}=-\frac{\left(D_{x x}-D_{y y}\right) / 2}{\sqrt{\left(D_{x x}-D_{y y}\right)^{2} / 4+D_{x y} \cdot D_{y x}}}, \\
& \sin \theta_{\vec{k}}=-\frac{D_{x y}}{\sqrt{\left(D_{x x}-D_{y y}\right)^{2} / 4+D_{x y} \cdot D_{y x}}} .
\end{aligned}
$$

All quantities in Eqs. 26 and 27 are those of a hexagonal WC in zero fields: $D_{x x}$ and $D_{y y}$ are the diagonal elements, $D_{x y}=D_{y x}$ the off-diagonal elements, of the dynamical matrix at $\vec{k}$; and $\omega_{T}^{0}$ and $\omega_{L}^{0}$ are the transverse and longitudinal phonon frequencies [62. We emphasize that this wavefunction is for distinguishable particles correlated within the lowest Landaulevel. 
In our variational calculations, we have allowed the coefficient of the overall exponent in Eq. 25, $A_{p}$, to vary from 1 . This is found to have only small effects on the total energies for $A_{p} \sim 1 \pm 0.2$. The optimal $A_{p}$ is found to be very close to 1 (see discussions below).

One comment is in order here. When we adopt the supercell geometry, the $\vec{k}$ 's in $B(\vec{k})$ are those compatible with the periodic boundary conditions. This is because the Fourier transform of $\xi_{i}$ in different simulation cells only has components at these selected $\vec{k}$ 's. Let us consider an arbitrary electron, say, electron 1 at $\vec{r}_{1}$ localized around a lattice site $\vec{R}_{1}$. Its motion is correlated with that of electron $\vec{r}_{2}$ localized around $\vec{R}_{2}$, in the form of $\xi_{1} B\left(\vec{R}_{1}, \vec{R}_{2}\right) \xi_{2}$. Due to the periodic boudary conditions, it is also correlated with all the images of $\vec{r}_{2}$ in a repeatative fashion. Physically, this correlation must take the form of $\xi_{1} B\left(\vec{R}_{1}, \vec{R}_{2}+\vec{T}\right) \xi_{2}$, not $\xi_{1} B\left(\vec{R}_{1}, \vec{R}_{2}\right)\left(\xi_{2}+\right.$ complex $\left.(\vec{T})\right)$. This procedure is precisely the same as that in Lam and Girvin's work 38 when they adopt the special $\vec{k}$-point sampling scheme to evaluate the energy and the wavefunction. Therefore, the magneto-phonon correlation factor is not periodic in $\vec{r}_{i}$, but rather in $\xi_{i}$. A technical point related to this physical requirement is that each electron must now be associated with a particular $\vec{T}$, in addition to an $\vec{R}$, if we wish to use this magneto-phonon correlation factor. In the magneto-phonon picture, all electrons are distinguishable, including those at different $\vec{R}$ 's or at different $\vec{T}$ 's. For two electrons within the same $\vec{T}$, we always evaluate the $\xi$ 's from the fixed $\vec{R}$ 's even though at a given step of the Monte Carlo walk, $\vec{r}_{1}$ may be closer to $\vec{R}_{2}$ than to $\vec{R}_{1}$. Same is true for two electrons with the same label 1 but lie in different $\vec{T}$ 's. This situation is entirely analogous to the evaluation of the total phonon zero-point-motion energy in a semiconductor, where all ions are distinguishable, but move in unison from one supercell to another.

The above correlation factor by construction does not mix in higher Landau-levels. We have also used a Landau-level-mixing correlation factor that has the same form as that in the absence of the magnetic field. For ease of reading and discussion, we rewrite it here:

$$
u(r)=\frac{A}{\sqrt{r}}\left(1-e^{-\sqrt{r / F}-\frac{1}{2} r / F}\right)
$$

where $r$ is the distance between the two electrons. When we adopt this correlation factor, 
electrons are not attached to specific lattice sites or to a particular simulation cell. We can therefore combine this correlation factor with the Slater determinant formed by one-particle orbitals in Eq. 24. The resultant wavefunction is one in which all electrons are identical regardless of the $\vec{R}$ 's and $\vec{T}$ 's that they happen to lie close to at any particular moment. This allows us to assess the effects of exchange quantitatively. The correlation factor in Eq. 28 mixes even and odd terms in the sense of phonon expansion of the Hamiltonian [37], and allows one to adjust the cusp-condition. In all the calculations reported below, we have used $A /\left(3 F^{3 / 2}\right)=1 / 3$. We have tested the cusp-condition with other values and found $A /\left(3 F^{3 / 2}\right)=1 / 3$ to be near the optimal in all cases. We give the details in the next section. We note that the Jastrow factor in Eq. 28 also modifies the intra-Landal-level correlation. This can be seen by projecting the wavefunction with Eq. 28 onto the lowest Landau-level subspace.

The evaluation of $\frac{1}{\sqrt{r}}$ in the Jastrow factor is done with the usual Ewald sum method, now in $2 \mathrm{D}$. Its various derivatives, needed in the kinetic energy calculation, are treated in the same way.

The magneto-phonon correlation factor is expected to be quite good for long-range corre-

lation effects. We also expect the $\frac{1}{\sqrt{r}}$-term to be reasonable for short and intermediate-range correlations. It is near optimal in the absence of the magnetic field, as can be seen from comparison with GFMC calculations (see Table I). The combined result of these two correlation factors interpolates smoothly between the strong-field and the weak-field limits. It is thus expected to yield an excellent correlated wavefunction for a Wigner crystal in a strong magnetic field.

\section{Quality of the variational wavefunction: comparison to fixed-phase quantum Monte Carlo results}

After our work [6] was published, another theoretical work appeared [9] in which an extension of the fixed-node Monte Carlo method to systems without time-reversal symmetry 
was applied to the 2D electron system in a strong magnetic field. We now compare our variational Monte Carlo results to their work. The "fixed-phase" method is, in principle, able to find the lowest possible energy for a given choice of the phase of the wavefunction. It should be noted that the phase of the wavefunction must be fixed for every point in the entire configuration space.

Two useful conclusions emerge from the comparison between the present variational Monte Carlo results and the fixed-phase diffusion Monte Carlo results:

I) By intentionally restricting the variational freedom in our trial wavefunction so that its phase is the same as that used in Ref. [9], the variational method is able to reproduce the diffusion Monte Carlo energy to within the accuracy of the published results.

II) As a result, the source of the relatively poor quality of the choice of the phase for the Wigner crystal wavefunction used in Ref. [9] becomes apparent: It is due to the fact that the magneto-phonon correlations are not sufficiently included by the choice of the phase. The energy of the Wigner crystals given by the fixed-phase diffusion Monte Carlo in Ref. [9] can be lowered by choosing a phase including the magneto-phonon correlations 63. However, in view of I), it is expected that the present variational Monte Carlo results for the WC including the magneto-phonon correlation effects are sufficient for calculating the FQHEWC phase boundary.

We now give the numerical details of the comparison. The phase of the WC wavefunction used in Ref. [9] will be the same as that used in this work if we set $A_{p}=0$, that is, if we turn off the magneto-phonon correlations intentionally. We do so, and then optimize the energy with respect to $\beta$ in the one-particle orbitals and $A$ in the $1 / \sqrt{r}$ part of the twoparticle correlation factor which does not change the phase of the wavefunction. We obtain for $r_{s}=20$ an energy of -0.0504 (in a.u.) at $\nu=1 / 3$ and -0.0518 at $\nu=1 / 5$. These are to be compared with an energy of $-0.0505(1)$ at $\nu=1 / 3$ and an energy of $-0.0518(1)$ at $\nu=1 / 5$ from the fixed-phase diffusion Monte Carlo reported in Ref. [9] for $r_{s}=20$ 64]. (See Table IX for our best energies at these filling factors for $r_{s}=20$.) 


\section{ENERGY OF A WIGNER CRYSTAL IN THE FQHE REGIME: RESULTS AND DISCUSSIONS}

In this section, we present the detailed calculated results and discuss their possible experimental implications. There are two experimentally relevant parameters that determine the energies of the phases involved here: the filling factor $\nu$ (related to the carrier density and the magnetic field as $\nu=2 l_{B}^{2} / r_{s}^{2}$ when $l_{B}$ and $r_{s}$ are in atomic units), and the electron density parameter $r_{s}$ (determined by the density, dielectric screening of the host media, and the carrier effective mass). They in turn determine the two relevant energy scales of the problem: the Landau-level spacing $\hbar \omega_{C}$ and the electron-electron interaction $E_{c}=e^{2} / \varepsilon d$ where $\pi d^{2}=1 / n$. Their ratio:

$$
\frac{E_{c}}{\hbar \omega_{C}}=\nu r_{s} / 2,
$$

provides a measure of the amount of Landau-level-mixing. For typical 2D electron systems, $r_{s} \sim 2$ in atomic units. But for the $p$-doped systems, it is $r_{s} \sim 25$, if $m^{*} \sim 0.6 m_{e}$ [60; and

$r_{s} \sim 13$, if $m^{*} \sim 0.3 m_{e}$ [22]. For the case of $p$-type doping, we therefore expect Landaulevel-mixing to play an important role in determining the energies.

There are two mechanisms by which the WC may lower its interaction energy by admitting higher Landau-level components in its wavefunction: as an inhomogeneous system, both its mean-field Hartree energy and the dynamic correlation energy can be lowered. The former may be done by allowing a charge distribution more localized than that given by the lowest Landau-level orbitals, and the latter by having a nonanalytic correlation term in the Jastrow factor. We have therefore allowed $\beta$ in Eq. 24 to increase and included the Landaulevel-mixing Jastrow factor in Eq. 28 in our final variational wavefunction, in addition to the magneto-phonon correlations contained in Eq. 26. Both mechanisms are found to be important for obtaining an optimal variational energy.

In Sec. IVA, we compare the present VMC results with previous lowest-Landau-level-only calculations. The energy from a single Slater determinant with $\beta \neq 1$ is given in Sec. IVB for 
$r_{s}=20$, and compared to available Hartree-Fock calculations also with Landau-level-mixing [36]. In Sec. IVC, we show that the finite size effects are negligible and quantify the effect of exchange in the present system. In Secs. IVD, IVE and IVF, we examine in some detail the effects of the various variational parameters in our trial wavefunction on the energy. In order to give a more general picture for the important effects in a Wigner crystal, we compare in Sec. IVG the WC energies using several different wavefunctions for $r_{s}=20$ and $\nu=1 / 3$ and $\nu=1 / 5$. Our calculations are brought into contact with the recent experiments in Sec. IVH where we compare the energy of a Wigner crystal to that of the FQHE liquid and derive a qualitative phase diagram. Finally, effects of finite temperatures and of disorder are discussed in Sec. IVI.

\section{A. Comparison to previous work within the lowest Landau-level approximation}

We first compare our results within the lowest Landau-level approximation to those obtained previously using different methods. We have constructed the "Hartree", exchangeonly, and correlation-only trial wavefunctions and evaluated the respective energies. The Hartree results correspond to those using a trial wavefunction that is simply a product of the

one-particle orbitals in Eq. 24 with $\beta=1$, with no Jastrow factors. These can be calculated exactly by the Ewald summation technique, and this fact is used as an independent check of the present VMC method. To include the exchange interaction, we use a Slater determinant trial wavefunction composed of the same one-particle orbitals as in the Hartree case. By "correlation-only", we mean the wavefunction given by Eq. 25 and Eq. 26.

The results from these calculations are given in Table $\mathbb{V}$ for $r_{s}=2.0$. The (interaction) energies at other $r_{s}$ 's may be obtained by simply scaling by $1 / r_{s}$ since we have implosed the lowest Landau-level approximation. The size of "bare" exchange energy may be estimated by comparing the Hartree and exchange-only results. In principle, an estimate of the size of exchange, "screened" by the magneto-phonon correlations, may be found by explicitly antisymmetrizing the wavefunction of Eq. 25. However, the resultant many-body wavefunc- 
tion is a sum of exponentially large number of terms, and cannot be used directly in an importance sampling calculation. Fortunately, the upper bound for exchange, set by the "bare" exchange interaction, is already very small for $\nu \leq \frac{1}{3}$. Landau-level-mixing decreases the exchange overlap still further, and provides additional screening. (We shall come back to this point later.) The kinetic energies for all these wavefunctions are explicitly evaluated and confirmed to be exactly $\frac{1}{2} \hbar \omega_{c}$ at every step of the Monte Carlo walks.

We note that our Hartree-Fock (exchange-only) calculation does not allow as much variational freedom as the previous ones which consider a charge-density wave state with the

order-parameters $\rho(\vec{G})$ being independent at different $\vec{G}$ 's [35]. But as can be seen from Table IV, this difference is not important for total energies. Our energies from the correlationonly calculation are also the same as those obtained by Lam and Girvin [38] using a special $k$-point sampling method. The remaining differences are within the quoted fitting errors [38. If we take the fractional quantum Hall liquid energies from Ref. 28, 34 that were obtained also within the lowest Landau-level approximation, Wigner crystallization occurs at $\nu_{c} \sim 1 / 6.5[38]$.

\section{B. Present results: single Slater determinant with Landau-level-mixing}

A calculation for the Wigner crystal that is both strictly variational and includes Landaulevel-mixing is done by MacDonald within the Hartree-Fock approximation [36. In order to compare with this calculation, we have calculated the Wigner crystal energy with a single Slater determinant. We simply seek the best one-particle orbital (optimizing $\beta$ in Eq. 24) in our VMC calculations.

We give the results for $r_{s}=20$ in Table $\nabla$ at $\nu=1 / 2, \nu=1 / 3, \nu=1 / 5$ and $\nu=1 / 7$. The optimal $\beta$ here also serves as a useful guide for later calculations involving correlations. The independent parameters in MacDonald's calculations [36] were chosen to be the filling factor $\nu$ and the energy ratio $\left(e^{2} / \varepsilon l_{B}\right) / \hbar \omega_{c}$ rather than $\nu$ and $r_{s}$ as in this work. At $\nu=1 / 2$ and $r_{s}=20$, the ratio $\left(e^{2} / \varepsilon l_{B}\right) / \hbar \omega_{c}$ is 10 , where he also reported his result. Converting his energy 
into the present atomic unit, (at $r_{s}=20$ and $\nu=1 / 2, e^{2} / \varepsilon l_{B}=0.1$ a.u., and $\hbar \omega_{c}=0.01$ a.u.) we find that his total energy (including the $\frac{1}{2} \hbar \omega_{c}$ ) is -0.04311 (his original result for the total energy was given without including the $\frac{1}{2} \hbar \omega_{c}$, and as a result is -0.04811 ), and his total electron-electron interaction energy is -0.05022 [36]. From the present calculations, we obtain $-0.0437(1)$ and $-0.0504(1)$, respectively. There is, of course, no variational principle for the interaction energy alone, and we do not assign a great significance to its value; however we can infer from these numbers that the amount of excess kinetic energy due to Landau-level-mixing from these two approaches is quite close. At $r_{s}=20$, it is probably insufficient to use only five lowest Landau-levels as was done in Ref. [36]. It appears that our single Slater determinant wavefunction is quite good in comparison with the self-consistent Hartree-Fock calculations.

If one ignores exchange, we then have a simple product of one-particle orbitals. The interaction energy for this "Hartree" state can be evaluated rapidly and accurately with the Ewald summation as we have done in the lowest Landau-level only cases. The kinetic energy for this state can also be obtained straightforwardly by projecting the one-particle orbitals (for example, take the one centered at $(0,0)$ ) onto the $|m\rangle-t h$ Landau-level which yields the following coefficient:

$$
c_{m}=\frac{2 \beta}{1+\beta^{2}}\left(\frac{1-\beta^{2}}{1+\beta^{2}}\right)^{m} .
$$

One can easily verify that $\sum_{0}^{\infty}\left|c_{m}\right|^{2}=1$. The projection also gives a rough measure of the size of Landau-level-mixing in cases involving correlations, although in the latter case projections can not be easily carried out. For illustration, we tabulated the values of $c_{m}$ for $\beta=1.3$ in Table $\nabla 1$.

\section{Finite size effects and the size of exchange}

The combination of the Ewald summation technique and the use of the image charges essentially eliminated the finite size dependence of the WC energy using modest size simulation cells. In Table VII, we give the finite size effects of the present VMC energies. The 
results given are for $r_{s}=20, \nu=1 / 3$, calculated with $\beta=1.12$ in the one-particle orbitals, magnotophonon correlation strenght $A_{p}=1.0$ (i.e., the original Lam-Girvin correlation factor), $A=10.0$ in the $\frac{1}{\sqrt{r}}$ correlation factor, $A / 3 F^{3 / 2}=1 / 3$, for cells of size $6 \times 6,6 \times 8$, $8 \times 8,8 \times 10,10 \times 10$, and $10 \times 12$.

As we mentioned above, a comparison of the Hartree and Hartree-Fock energies in Table IV provides a rough estimate of the size of the bare exchange. It is clear that for $\nu \leq 1 / 5$, we may safely ignore the exchange contribution to the total energy. We now discuss the size of the exchange contribution at $\nu=1 / 3$ near the calculated FQHE liquid-WC transition in more detail.

Both intra- and inter-Landau-level correlations can screen the exchange interaction. The latter favors smaller one-particle wavefunctions for a lower direct energy. This reduces the one-particle wavefunction overlap drastically. We estimate the size of the exchange interaction in the $\mathrm{WC}$ in the following way. We form two wavefunctions. One is a totally antisymmetric wavefunction formed with the product of the correlation factor in Eq. 28 with a Slater determinant of one-particle orbitals from Eq. 24; the other one has the same correlation factor, but now has only one term of the Slater determinant. We shall refer to the former loosely as "screened HF", and the latter "screened Hartree". Results are given in Table VIII for $r_{s}=20$ and $\nu=1 / 3$, where for comparison the unscreened ("bare") lowest-Landau-level-only results are listed again. The "screened" results are calculated with $\beta=1.2$ and $A=10$. The energies from the "screened" wavefunctions are the same to within the statistical noise. The magneto-phonon correlation will further screen the exchange interaction. We thus conclude that exchange in the Wigner crystal phase is not important for its energy.

\section{Optimal cusp-condition and the magneto-phonon correlation}

We have also varied the cusp-conditions given by $A / 3 F^{3 / 2}$ and the strength of the magneto-phonon correlation factor $A_{p}$. The cusp-condition derived from the equation of 
motion of two particles in a strong magnetic field is different from that without the magnetic field. We have considered the short (relative) distance behavior of two electrons in a strong magnetic field. We find that for an eigenstate with relative angular momentum $m$, the cusp-condition associated with the divergence of the Coulomb interaction is:

$$
\frac{A}{3 F^{3 / 2}}=\frac{\nu}{\nu+2}
$$

However, we have checked that changing the cusp-condition at a fixed $A$ from the value of $A / 3 F^{3 / 2}=1 / 3$ by to up 50 percent at $\nu=1 / 3,1 / 5$ and $r_{s}=20$ does not affect the energy. This presumably results from the strong localization of the single-particle orbitals involved. As a result, the particles are not too close to each other; and the short range cusp-conditions are not important. For example, at $\nu=1 / 5$ and $r_{s}=20$, for $\beta=1.04$, $A_{p}=1$, and $A=10$, with $A / 3 F^{3 / 2}=1 / 3$, the total energy (with $\frac{1}{2} \hbar \omega_{c}$ ) is $-0.03962(1)$ a.u., and with $A / 3 F^{3 / 2}=1 / 5.5$, it is $-0.03961(1)$ a.u.

In Fig. 6, we show the total energy as a function of the strength of the magneto-phonon correlation factor $A_{p}$ for $r_{s}=20$ and $\nu=1 / 3$. Here we have set $A=0$. The energy changes relatively little and the optimum occurs for $A_{p}=1$. This is not surprising since $A_{p}=1$ is the desired value for asymptotically small $\nu$. Calculations are also done for other filling factors and the conclusion remains.

\section{E. Effects of $\beta$ on Wigner crystal energy}

We find that for the $r_{s}$ and $\nu$ values relevant to the recent experiments carried out on p-doped samples [22], squeezing the one-particle wavefunction is the single most important mechanism for lowering the energy of a WC [6]. To focus on its effects on the total energy, we can set $A_{p}=1$ and $A=0$ and calculate the total energy as a function of $\beta$. This was doen in Figs. 1(a) and 1(b) of Ref. [6], where we showed the total energy as a function of $\beta$ at $\nu=1 / 3$ for $r_{s}=2$ and $r_{s}=20$ respectively. When $\beta$ is optimized, the density at the lattice sites increases by $\delta \rho(0) / \rho(0)=70 \%$ and the energy is lowered by $\delta E /\left(E-\frac{1}{2} \hbar \omega_{C}\right)=-4.4 \%$ 
at $r_{s}=20$. The changes are respectively $10 \%$ and $-0.8 \%$ at $r_{s}=2$. Fig. 7 and Fig. 8 show how the kinetic energy and the potential energy change with $\beta$ for $r_{s}=20$ and $\nu=1 / 3$. At the optimal $\beta=1.3$, the kinetic energy has risen only by $\sim 0.001 a . u$. while the potential energy gain is 0.0034 a.u. compared to $\beta=1$. For $\beta$ greater than 1.3 , a more rapid rise in kinetic energy than the drop in potential energy makes it less favorable, although even at $\beta=1.6$, the total energy is still lower than that at $\beta=1$. For comparison, we also give the energy at $r_{s}=20$ and $\nu=1 / 5$ as a function of $\beta$ in Fig. 9. The optimal $\beta$ is around 1.1 and the energy gain is much smaller than that at $\nu=1 / 3$.

We now come back to the case of $r_{s}=20$ and $\nu=1 / 3$. A $4 \%$ lowering in energy obtained by changing $\beta$ alone is extremely important for determining the phase boudary between the FQHE liquid and the WC. To see this, we note that the interaction energy of the lowest Landau-level Lam-Girvin wavefunction is only $12 \%$ higher than the absolute minimum set by the Ewald energy. At $r_{s}=20$, this difference is reduced by one-third, by allowing the Landau-levels to mix through the one-particle orbitals. In comparison to the size of the correlation and Landau-level-mixing effects, the exchange contributions to the total energies are indeed negligible.

\section{F. Effects of $A$ and the continuity of $E(\nu)$}

In Fig. 10, we show the total energy of the $\mathrm{WC}$ as a function of $A$, the coefficient of the $1 / \sqrt{r}$-term in the Jastrow factor. The results are calculated for the case of $r_{s}=20, \nu=1 / 3$ with $A_{p}=1$ and $\beta=1.17$.

We have examined the continuity of the WC energy as a function of filling factor $\nu$ using the VMC method. We first calculated the WC energies for two filling factors on both sides of $\nu=1 / 3$. Within the present WC wavefunction and the numerical accuracy, there is no cusp-like feature occurring at $\nu=1 / 3$. We also studied the WC energy at $\nu=9 / 20,1 / 2$, and $11 / 20$. Again, we find no sign of any discontinuity at $\nu=1 / 2$ within the Wigner crystal wavefunctions. We therefore conclude that the solid energy curve is continuous for all filling 
factors, showing no features at either even- or odd-denominator filling factors.

\section{G. Energies of a Wigner crystal: comparison of different wavefunctions}

In Fig. 11 and Fig. 12, we show, for $r_{s}=20, \nu=1 / 3$, and $r_{s}=20, \nu=1 / 5$, the VMC energies for a Wigner crystal using the various different wavefunctions discussed. These two figures show quite clearly which components of the electron correlations are important and how they vary with filling factor. For comparison, the energies of the corresponding Laughlin liquid with and without Landau-level-mixing from Refs. [7, 34] are also given. For $\nu=1 / 3$, the largest energy gain occurs when we allow the Gaussian size to decrease while keeping the magneto-phonon correlation. Further including the $\frac{1}{\sqrt{r}}$ correlation factor given by Eq. 28 introduces a relatively small lowering of the energy. We point out that the optimal single-particle orbital Gaussian size is larger when the $1 / \sqrt{r}$ correlation factor is included in the wavefunction. With all the correlation effects taken into account, our solid energy still lies above that of the liquid (with Landau-level-mixing effects) at $r_{s}=20$. Crystallization does not occur until a larger $r_{s}$ at $\nu=1 / 3$.

At $\nu=1 / 5$, the largest contribution now comes from intra-Landau-level correlation effects, but still Landau-level-mixing correlations are comparable in size. Without Landaulevel-mixing effects, the solid energy lies above that of the liquid. But with Landau-levelmixing effects, the solid becomes lower in energy by an amount that is significant on the scale of energy differences in the present context. We therefore conclude that for $\nu=1 / 5$ at $r_{s}=20$, the solid is lower in energy than the FQHE liquid. At even smaller filling factors, the intra-Landau-level effects are by far the most important effect, and we find that the Wigner crystal is more stable in energy (see, however, Ref. [25]). 


\section{H. Wigner crystal versus FQHE liquid: ground-state energies and the general phase diagram}

In obtaining our final results for the energy of a Wigner crystal, we have optimized both $\beta$ and $A$ at a given $r_{s}$ and $\nu$. Optimization of $A_{p}$ is inconsequential for the total energy. Results for $r_{s}=20$ are plotted in Fig. 13 as solid lines. The energies from the magnetophonon correlated wavefunction with no Landau-level-mixing are shown as empty squares. The energies from the correlated wavefunction with Landau-level-mixing are shown as filled squares. They are obtained by varying $\beta$ in the one-particle orbitals and $A$ in the $1 / \sqrt{r}$ Jastrow factor while keeping $A_{p}=1$ in the magneto-phonon correlation factor. The energies of the incompressible FQHE liquid are shown as dotted lines. Both the lowest-Landaulevel results [34 based on Laughlin's variational wavefunction, and the recent results with Landau-level-mixing [7] are plotted. For the liquid state, the actual calculated energies at $\nu=1 / 3,1 / 5$, and $1 / 7$ are plotted as hexagons in Fig. 13. The lines passing through them are a spline fit to the data. They do not show the cusps that must occur at filling factors where the FQHE states exist. On the other hand, the energy of the solid is considered to be valid for all filling factors due to its continuity discussed in Sec. IVF.

In general, it is expected that Landau-level-mixing effects will be smaller in the liquid phase. As discussed in beginning of this section, in the solid phase, both the Hartree and correlation energies can be lowered by allowing Landau-level-mixing. The former mechanism is found to be more important for lowering the energy in WC, but it is entirely lost in the uniform liquid phase whose Hartree energy will not be altered by Landau-level-mixing. This expectation is confirmed by the work of Price, Platzman and He [7]. They find that the lowering in the liquid energy is indeed substantially smaller than that in the solid: for $r_{s}=20$ at $\nu=1 / 3$, lowering in energy from Landau-level-mixing for the FQHE liquid state is only 1/4 of that we find for the WC solid (see Fig. 11 and Fig. 13). As a result, for $r_{s}=20$, the FQHE state with Landau-level-mixing is only slightly more stable than the WC state at $\nu=1 / 3$ for a pure system with no disorder. At $\nu=1 / 5$, the WC becomes lower in energy. 
In Table $\llbracket \mathbb{X}$, we list the optimized energies for the Wigner crystal and the FQHE liquid for $r_{s}=20$ at several filling factors. For $r_{s}=2$, the WC state is higher in energy at $\nu=1 / 5$ but remains lower in energy at $\nu=1 / 7$ than the FQHE state.

Based on these theoretical results, we present a qualitative phase diagram for the 2D electron gas in a strong magnetic field. In Fig. 14, the $x$-axis is the Landau-level filling factor and the $y$-axis is $r_{s}$ measured in effective atomic units which may be changed by the carrier effective mass at a given doping concentration. At a small enough filling factor or low enough density, the system crystallizes. At intermediate experimental parameters, a number of reentrant phase transitions at $\nu=1 / 5, \nu=1 / 3, \nu=1$, etc, are expected as one scans the magnetic field. But at a given filling factor, we only expect one phase transition as $r_{s}$ is varied. The picture is only meant to be illustrative; details such as the strength of various phases (peak heights in the figure) should not be taken literally. The details of the phase diagram will be affected by temperature and disorder, both of which to lowest order favor the Wigner crystal phase. We discuss qualitatively these two issues in the next subsection.

\section{Effects of disorder and finite temperatures}

Our calculations are carried out for a perfect 2D electron gas with no disorder and at zero temperature. Real systems do not satisfy either condition. Due to the reduced dimentionality, both are expected to have important effects on the phase diagram of the system.

The presence of disorder breaks the Wigner crystal into domains of a finite linear size $\xi^{T}$ [65]. The superscript on $\xi$ is intended to indicate that most of the static distortions in a WC are transverse [65. The Wigner crystal can lower its energy by locally adjusting its density to accommodate for the local disorder potential. The quantum Hall liquid on the other hand is rigid to disturbances on energy scales smaller than the gap in its collective excitations. Therefore to first order, the presence of disorder favors the Wigner crystal formation. The

margin by which the Wigner crystal is favored due to disorder has been estimated [7,66] 
using $\xi$ deduced from nonlinear transport threshold field experiments [14, 17, 18,21, 65]. Such a procedure, while suggestive, is not very accurate. Quantitatively, the disorder affects the energy differences between the solid and the liquid, but the qualitative picture presented above is found not to be altered [66] by such estimates. The precise pinning mechanism of the Wigner crystal by disorder in the actual experimental GaAs/AlGaAS heterojunction systems is at present not clear, and it remains a subject of considerable amount of experimental and theoretical interest 67.

The effects of finite temperatures on either the Wigner crystal or the quantum Hall liquid are a difficult issue. On the fractional quantum Hall liquid side, while it is often thought that there is only a gradual decay of the peculiar FQHE order with rising temperature [71], some recent experiments point to the possibility of a finite temperature transition that is in fact rather abrupt 20]. There is currently no theoretical model that can account for this observation. On the Wigner crystal side, a classical solid in 2D is expected to melt by the Kosterlitz-Thouless mechanism as the temperature increases [68]. A two-step melting path has been suggested [69]. The present system has significant quantum mechanical fluctuation effects and it is unclear if the classical $2 \mathrm{D}$ melting theory is applicable in the vicinity of a quantum phase transition to the FQHE state.

However, at temperatures well below the afore-mentioned phase transition temperatures, the collective excitations in both the solid and the liquid may be approximated as independent bosons [25,41. Only the lower branch needs to be considered, i.e., the magneto-roton for the FQHE liquid and the (largely transverse) magneto-phonon for the Wigner crystal. In this regime, one can then evaluate, and compare, the free energies of these two phases, and determine a finite temperature phase diagram [25],66]. While some interesting effects have been predicted, it remains unclear if these effects lie outside the regime of validity of the low-temperature assumption. The presence of the disorder pinning gap in the solid phase may also affect the entropy of the Wigner crystal. Further theoretical efforts are needed in these directions.

Finally, we note that there have been recent claims that Wigner crystallization occurs 
for 2D electron gas at doped $\mathrm{Si} / \mathrm{SiO}_{2}$ interfaces at low or zero external magnetic fields [23]. The reentrant insulating phases set in around $\nu=1$ (and $\nu=2$ ) in these systems. We have carried out calculations to determine the Wigner crystal/quantum Hall liquid phase boundary at $\nu=1$ and found that the resultant density is much lower than the experimental doping concentration reported in these works [23]. The band structure of Si around the conduction band minimum is more complicated than that of $\mathrm{GaAs}$ [23]. It is doubtful if the present model of a one-component fermion system is capable of describing the $\mathrm{Si}_{\mathrm{SiO}}$ system. Theoretically, it has been suggested that multi-component systems are more susceptible to Wigner crystal or charge-density-wave instabilities since they may arrange their density profiles to be mutually beneficial energetically [70], but this simple picture may not be correct when inter-layer interaction induced frustration of the triangular lattice is important. A variational Monte Carlo calculation has recently been carried out for the related phenomenon of Wigner crystallization in a wider quantum well, but within the lowest subband approximation [72]; generalizations of this approach to double layer systems would be interesting. While it is possible that such mechanisms are indeed responsible for the insulating phases observed, the much lower mobility of the $S i$ samples [23], as compared to the GaAs samples [21], also brings to mind the possible role of disorder in the insulating phase.

\section{SUMMARY}

In summary, we have studied variationally the ground-state energies of two-dimensional electron Wigner crystals, both with and without an external magnetic field. We identify the important quantum fluctuations present in an optimal ground-state wavefunction. We take into account both the short-range and long-range correlation effects in the $\mathrm{WC}$ and provide a rigorous upper bound for the WC energy. Landau-level-mixing effects are shown to be significant in the range of carrier density, effective mass, and strength of the magnetic field

of experimental interest. In the context of the experimentally observed insulating phases in 
the fractional quantum Hall effect regime, our results strongly suggest that the main driving force of the phase transitions is electron-electron interaction in the best current samples. More work is needed to further understand the effects of finite temperatures and of disorder. This will probably require a better understanding of both the neutral and charged excitations of this interesting electron solid.

\section{ACKNOWLEDGMENTS}

We thank Drs. Sankar Das Sarma, Song He, Pui Lam, Peter Littlewood, Andy Millis, Phil Platzman, Rod Price, and Andrew Rappe for discussions and collaborations on related subjects. Work at Berkeley was supported by NSF through Grant No. DMR91-20269 and by DOE through Contract No. DE-AC03-76SF00098. CRAY computer time was provided by the NSF at the San Diego Supercomputing Center and by the DOE. Work at Rutgers was supported by NSF through DMR92-21907. 


\section{REFERENCES}

[1] E. P. Wigner, Phys. Rev. 46, 1002 (1934); and Trans. Faraday Soc. 34, 678 (1938).

[2] W. L. McMillan, Phys. Rev. 138, A442 (1965).

[3] D. Ceperley and B. Alder, Nature 231, 555 (1986); Phys. Rev. Lett. 45, 566 (1980).

[4] S. Fahy, X. W. Wang, and S. G. Louie, Phys. Rev. B 42, 3503 (1990).

[5] D. Ceperley, Phys. Rev. B 18, 3126 (1978); B. Tanatar and D. M. Ceperley, Phys. Rev. B 39, 5005 (1989).

[6] X. J. Zhu and S. G. Louie, Phys. Rev. Lett. 70, 335 (1993).

[7] R. Price, P. M. Platzman, and S. He, Phys. Rev. Lett. 70, 339 (1993).

[8] A. M. Rappe, X. J. Zhu, and S. G. Louie, unpublished.

[9] G. Ortiz, R. M. Martin, and D. M. Ceperley, Phys. Rev. Lett. 71, 2777 (1993).

[10] D. C. Tsui, H. L. Stormer, and A. C. Gossard, Phys. Rev. Lett. 48, 1559 (1982).

[11] The Quantum Hall Effect, 2nd. ed., eds. R. E. Prange and S. M. Girvin (Springer-Verlag, New York, 1990).

[12] B. I. Halperin, Helv. Phys. Acta 56, 75 (1983).

[13] H. W. Jiang, R. L. Willett, H. L. Stormer, D. C. Tsui, L. N. Pfeiffer, and K. W. West, Phys. Rev. Lett. 65, 633 (1990).

[14] V. J. Goldman, M. Santos, M. Shayegan, J. E. Cunningham, Phys. Rev. Lett. 65, 2189 (1990).

[15] E. Y. Andrei, G. Deville, D. C. Glattli, F. I. B. Williams, E. Paris, and B. Etienne, Phys. Rev. Lett. 60, 2765 (1988); E. Y. Andrei, F. Perruchot, and F. I. B. Williams, unpublished; H. L. Stormer and R. L. Willett, Phys. Rev. Lett. 62, 972 (1989). 
[16] M. A. Paalanen, R. L. Willett, P. B. Littlewood, R. R. Ruel, K. W. West, L. N. Pfeiffer, and D. J. Bishop, Phys. Rev. B 45, 11342 (1992); for a recent review, see, R. L. Willett, Surf. Sci. 305, 76 (1994).

[17] F. I. B. Williams, P. A. Wright, R. G. Clark, E. Y. Andrei, G. Deville, D. C. Glattli, O. Probst, B. Etienne, C. Dorin, C. T. Foxon, and J. J. Harris, Phys. Rev. Lett. 66, 3285 (1991); H. L. Stormer and R. L Willett, Phys. Rev. Lett. 68, 2104 (1992);

[18] Y. P. Li, T. Sajoto, L. W. Engel, D. C. Tsui, and M. Shayegan, Phys. Rev. Lett. 67, 1630 (1991); and Y. P. Li, D. C. Tsui, T. Sajoto, L. W. Engel, M. Santos, and M. Shayegan, unpublished.

[19] H. Buhmann, et al., Phys. Rev. Lett. 66, 926 (1991); ibid. 65, 1056 (1990); I. V. Kukushkin, et al., Europhys. Lett. 23, 211 (1993); I. V. Kukushkin, et al., Phys. Rev. Lett. 72, 3594 (1994).

[20] I. V. Kukushkin, et al., Europhys. Lett. 22, 287 (1993); ibid. 18, 63 (1992).

[21] H. W. Jiang, H. L. Stormer, D. C. Tsui, L. N. Pfeiffer, and K. W. West, Phys. Rev. B 44, 8107 (1991).

[22] M. B. Santos, Y. W. Suen, M. Shayegan, Y. P. Li, L. W. Engel, and D. C. Tsui, Phys. Rev. Lett. 68, 1188 (1992); M. B. Santos, J. Jo, Y. W. Suen, L. W. Engel, and M. Shayegan, Phys. Rev. B 46, 13639 (1992); H. C. Manoharan and M. Shayegan, unpublished.

[23] V. M. Pudalov, M. D'Iorio, S. V. Kravchenko, and J. W. Campbell, Phys. Rev. Lett. 70, 1866 (1993); M. D’Iorio, V. M. Pudalov, and S. G. Semenchinsky, Phys. Rev. B 46, $15992(1992)$.

[24] S. Kivelson, D. H. Lee, and S. C. Zhang, Phys. Rev. B 46, 2223 (1992); B. I. Halperin, P. A. Lee, N. Reed, Phys. Rev. B 47, 7312 (1993). 
[25] P. M. Platzman and R. Price, Phys. Rev. Lett. 70, 3487 (1993); R. Price, X. J. Zhu, P. M. Platzman, and S. G. Louie, Phys. Rev. B 48, 11473 (1993).

[26] C. C. Grimes and G. Adams, Phys. Rev. Lett. 42, 795 (1979); D. S. Fisher, B. I. Halperin, and P. M. Platzman, ibid., 798 (1979).

[27] T. F. Rosenbaum, S. B. Field, D. A. Nelson, and P. B. Littlewood, Phys. Rev. Lett. 54, $241(1985)$.

[28] R. B. Laughlin, Phys. Rev. Lett. 50, 1395 (1983).

[29] A. V. Chaplik, Sov. Phys. JETP 35, 395 (1972). [Zh. Eksp. Teor. Fiz. 62, 746 (1972)]

[30] C. M. Care and N. H. March, Adv. Phys. 24, 101 (1975); and references therein.

[31] X. J. Zhu and S. G. Louie, unpublished.

[32] X. J. Zhu and S. G. Louie, Phys. Rev. B 48, 13661 (1993).

[33] F. D. M. Haldane and E. H. Rezayi, Phys. Rev. Lett. 54, 237 (1985); F. D. M. Haldane, Ch. 8 in Ref. [11]

[34] D. Levesque, J. J. Weis, and A. H. MacDonald, Phys. Rev. B 30, 1056 (1984).

[35] D. Yoshioka and H. Fukuyama, J. Phys. Soc. Jpn. 47, 394 (1979); D. Yoshioka and P. A. Lee, Phys. Rev. B 27, 4986 (1983); K. Maki and X. Zotos, Phys. Rev. B 28, 4349 (1983).

[36] A. H. MacDonald, Phys. Rev. B 30, 4392 (1984).

[37] K. Esfarjani and S. T. Chui, J. Phys. C 3, 5825 (1991); S. T. Chui, T. M. Hakim, and K. B. Ma, Phys. Rev. B 33, 7110 (1986).

[38] P. K. Lam and S. M. Girvin, Phys. Rev. B 30, 473 (1984); 31, 613 (E) (1985).

[39] K. Esfarjani and S. T. Chui, Phys. Rev. B 42, 10758 (1990). 
[40] B. I. Halperin, Phys. Rev. Lett. 52, 1583 (1984); (E) 52, 2390 (1984).

[41] S. M. Girvin, A. H. MacDonald, and P. M. Platzman, Phys. Rev. Lett. 54, 581 (1985); Phys. Rev. B 33, 2481 (1986).

[42] For a review on CDW systems, see, G. Grüner, Rev. Mod. Phys. 60, 1129 (1988).

[43] X. J. Zhu, P. B. Littlewood, and A. J. Millis, Phys. Rev. Lett. 72, 2255 (1994); and Phys. Rev. B 50, 4600 (1994).

[44] A. Pinczuk, B. S. Dennis, L. N. Pfeiffer, K. West, Phys. Rev. Lett. 70, 3983 (1993); L. L. Sohn, A. Pinczuk, B. S. Dennis, L. N. Pfeiffer, K. W. West, and L. Brey, unpublished.

[45] D. J. Thouless, Proc. Phys. Soc. London 86, 893 (1965).

[46] M. Roger, J. H. Hetherington, and J. M. Delrieu, Rev. Mod. Phys. 55, 1 (1983); M. Roger, Phys. Rev. B 30, 6432 (1984); M. Roger, Phys. Rev. Lett. 64, 297 (1990).

[47] C. Herring, Chap. 4 in Magnetism, eds. C. I. Rado and H. Suhl, Vol. IV, (Academic Press, London, 1966).

[48] S. Kivelson, C. Kallin, D. P. Arovas, J. R. Schrieffer, Phys. Rev. Lett. 56, 873 (1986); Phys. Rev. B 36, 1620 (1987).

[49] G. Baskaran, Phys. Rev. Lett. 56, 2716 (1986); D. H. Lee, G. Baskaran, and S. Kivelson, Phys. Rev. Lett. 59, 2467 (1987).

[50] D. J. Thouless and Q. Li, Phys. Rev. B 36, 4581 (1987).

[51] S. A. Kivelson, C. Kallin, D. P. Arovas, and J. R. Schrieffer, Phys. Rev. B 37, 9085 (1988).

[52] D. M. Ceperley and G. Jacucci, Phys. Rev. Lett. 58, 1648 (1987).

[53] A. K. McMahan and J. W. Wilkins, Phys. Rev. Lett. 35, 376 (1975).

[54] See, e.g., R. R. P. Singh and D. A. Huse, Phys. Rev. Lett. 68, 1766 (1992); and references 
therein.

[55] T. Kato, Comm. Pure and App. Math. 10, 151 (1957); C. R. Myers, C. J. Umrigar, J. P. Sethna, J. D. Morgan, Phys. Rev. A 44, 5537 (1991).

[56] X. C. Xie, S. He, and S. Das Sarma, Phys. Rev. Lett. 66, 389 (1991).

[57] R. P. Feynman, Statistical Mechanics, (Addison-Wesley, New York, 1972).

[58] S. F. Edwards, Proc. Phys. Soc. 72, 685 (1958); T. Gaskell, ibid. 77, 1182 (1961); M. A. Pokrant and F. A. Stevens, Jr., Phys. Rev. A 7, 1630 (1973).

[59] D. Yoshioka, J. Phys. Soc. Jpn. 53, 3740 (1984); 55, 885 (1986).

[60] H. L. Stormer, Z. Schlesinger, A. Chang, D. C. Tsui, A. C. Gossard, and W. Wiegmann, Phys. Rev. Lett. 51, 126 (1983); R. M. Hannak and W. W. Rühle, Phys. Rev. B 50, 15445 (1994).

[61] J. Zak, Phys. Rev. 134, A1602 (1964); A1607 (1964).

[62] L. Bonsall and A. A. Maradudin, Phys. Rev. B 15, 1959 (1977); for a more recent quantum mechanical treatment within the Hartree-Fock approximation, see, R. Cote and A. H. MacDonald, Phys. Rev. B 44, 8759 (1991).

[63] In order to do so, the electrons must now be treated as distinguishable particles as we discussed in the previous subsection, Sec. IIID. This however has little effect on the WC total energy, see Sec. IVC below for a detailed quantitative exposition.

[64] The uncertainties in the fixed-phase results are from the fact that they are read off from Fig. 3 in Ref. [9]. The energies here do not include the term of $\frac{1}{2} \hbar \omega_{c}$.

[65] H. Fukuyama and P. A. Lee, Phys. Rev. B 17, 535 (1978); P. A. Lee and H. Fukuyama, ibid., 542 (1978); P. A. Lee and T. M. Rice, ibid. 20, 1345 (1979); B. G. A. Normand, P. B. Littlewood, and A. J. Millis, ibid. 46, 3920 (1992). 
[66] R. Price, P. M. Platzman, S. He, and X. J. Zhu, Surf. Sci. 305, 126 (1994); and P. M. Platzman and R. Price, unpublished.

[67] I. M. Zuzin, S. Marianer, and B. I. Shklovskii, Phys. Rev. B 46, 3999 (1992); M. C. Cha and H. A. Fertig, Phys. Rev. B 50, 14368 (1994); for some experimental discussions on the possible pinning mechanisms, see Ref. [18].

[68] J. M. Kosterlitz and D. J. Thouless, J. Phys. C 6, 1181 (1973).

[69] A. P. Young, Phys. Rev. B 19, 1855 (1979); D. R. Nelson and B. I. Halperin, ibid., 2457 (1979).

[70] L. Swierkowski, D. Neilson, and J. Szymanski, Phys. Rev. Lett. 67, 240 (1991); L. Zhang and H. A. Fertig, unpublished; S. Narasimhan and T. L. Ho, unpublished.

[71] E. H. Rezayi and F. D. M. Haldane, Phys. Rev. Lett. 61, 1985 (1988).

[72] R. Price, X. J. Zhu, S. Das Sarma, and P. M. Platzman, Phys. Rev. B 51, 2017 (1995). 


\section{TABLES}

TABLE I. Energy/electron (in $10^{-3}$ atomic units) of the Wigner crystal in a $2 \mathrm{D}$ hexagonal lattice from the present VMC calculation in comparison with previous work. Results are for a simulation cell of 56 electrons.

\begin{tabular}{lccc}
\hline \hline & $r_{s}=30$ & $r_{s}=50$ & $r_{s}=100$ \\
\hline VMC-Present & $-31.83(1)$ & $-19.82(1)$ & $-10.23(1)$ \\
$\mathrm{VMC}^{a}$ & $-31.82(1)$ & $-19.79(1)$ & $-10.24(1)$ \\
& & & $-10.24(1)$ \\
$\mathrm{GFMC}^{a}$ & $-31.89(1)$ & $-19.81(1)$ & \\
\hline \hline
\end{tabular}

a Ref. [5]

TABLE II. Energy/electron (in $10^{-3}$ atomic units) for electrons in hexagonal (HX), square (SQ), and honeycomb (HC) lattices. Results are for the spin-polarized state. Sizes of the simulation cells are slightly different for each lattice and in every case the resulting finite size effects are smaller than the statistical noise (see Table III).

\begin{tabular}{lcccc}
\hline \hline & $r_{s}=30$ & $r_{s}=50$ & $r_{s}=70$ & $r_{s}=100$ \\
\hline $\mathrm{HX}$ & $-31.83(1)$ & $-19.82(1)$ & $-14.38(1)$ & $-10.23(1)$ \\
$\mathrm{SQ}$ & $-31.72(1)$ & $-19.71(1)$ & $-14.34(1)$ & $-10.19(1)$ \\
$\mathrm{HC}$ & $-31.52(1)$ & $-19.60(1)$ & $-14.25(1)$ & $-10.13(1)$ \\
\hline \hline
\end{tabular}


TABLE III. Finite size effects (energy/electron in $10^{-3}$ atomic units) for electrons in the honeycomb (HC) lattice and the square (SQ) lattice at $r_{s}=30$.

\begin{tabular}{|c|c|c|c|}
\hline & $\mathrm{N}=32$ & $\mathrm{~N}=50$ & $\mathrm{~N}=72$ \\
\hline \multicolumn{4}{|c|}{ honeycomb } \\
\hline $\mathrm{FM}$ & $-31.50(1)$ & $-31.51(1)$ & $-31.51(1)$ \\
\hline \multirow[t]{2}{*}{ AFM } & $-31.67(1)$ & $-31.67(1)$ & $-31.67(1)$ \\
\hline & $\mathrm{N}=36$ & $\mathrm{~N}=48$ & $\mathrm{~N}=64$ \\
\hline \multicolumn{4}{|c|}{ square } \\
\hline FM & $-31.71(1)$ & $-31.72(1)$ & $-31.72(1)$ \\
\hline $\mathrm{AFM}$ & $-31.67(1)$ & $-31.67(1)$ & $-31.67(1)$ \\
\hline
\end{tabular}

TABLE IV. Energies (in effective atomic units) of the hexagonal Wigner crystal from various lowest Landau-level only calculations at $r_{s}=2.0$. A constant kinetic energy $\frac{1}{2} \hbar \omega_{C}$ is subtracted.

\begin{tabular}{|c|c|c|c|c|c|c|}
\hline \multirow[t]{2}{*}{$\nu$} & \multirow[t]{2}{*}{ Hartree } & \multicolumn{2}{|c|}{ Exchange-only } & \multicolumn{2}{|c|}{ Correlation-only } & \multirow{2}{*}{$\begin{array}{l}\text { Laughlin } \\
\text { Ref. 34 }\end{array}$} \\
\hline & & Present & Ref. [35, 38] & Present & Ref. 38 & \\
\hline $1 / 2$ & -0.4222 & $-0.4435(3)$ & -0.4438 & $-0.4397(13)$ & -0.4396 & \\
\hline $1 / 3$ & -0.4722 & $-0.4762(8)$ & -0.4758 & $-0.4834(9)$ & -0.4836 & -0.5023 \\
\hline $1 / 4$ & -0.4960 & $-0.4966(5)$ & -0.4957 & $-0.5040(3)$ & -0.5034 & \\
\hline $1 / 5$ & -0.5090 & $-0.5091(1)$ & -0.5090 & $-0.5155(3)$ & -0.5151 & -0.5180 \\
\hline $1 / 7$ & -0.5226 & $-0.5225(1)$ & -0.5220 & $-0.5272(1)$ & -0.5264 & -0.5256 \\
\hline
\end{tabular}


TABLE V. Wigner crystal energy (in atomic units per electron) for a single Slater determinant with one-particle orbitals given in Eq. 18, for $r_{s}=20$. The optimal variational parameter $\beta$ at which these calculations are done is also given.

\begin{tabular}{cccr}
\hline \hline Filling factor $\nu$ & $\beta$ & Total Energy & $\frac{1}{2} \hbar \omega_{c}$ \\
\hline $1 / 2$ & 1.5 & $-0.0437(1)$ & 0.0050 \\
$1 / 3$ & 1.37 & $-0.0424(1)$ & 0.0075 \\
$1 / 5$ & 1.15 & $-0.0391(1)$ & 0.0125 \\
$1 / 7$ & 1.075 & $-0.0350(1)$ & 0.0175 \\
\hline \hline
\end{tabular}

TABLE VI. Overlap of $\phi(\beta)$ in Eq. 18 with the $n t h$-LL: $\langle$ nth $L L| \phi(\beta)>$ with $\beta=1.3$. This gives a measure of the amount of Landau-level-mixing in the ground-state wavefunction corresponding to a Wigner crystal at $r_{s}=20$ and $\nu=1 / 3$ (see text).

\begin{tabular}{llll}
\hline \hline$n=0$ & $n=1$ & $n=2$ & $n=3$ \\
\hline 0.9666 & -0.2480 & 0.0636 & -0.0163 \\
\hline \hline
\end{tabular}


TABLE VII. Finite size effects and statistical noise in this work have been reduced to a level that is unimportant for the energy differences of interest. We show a typical example for $\nu=1 / 3$, $r_{s}=20$, calculated with $A=10.0, A_{p}=1.0, A /\left(3 F^{3 / 2}\right)=1 / 3, \beta=1.12$. See text for these parameters in the wavefunction. The total energies here are in atomic units and include the term $\frac{1}{2} \hbar \omega_{c}=0.0075$ a.u.

\begin{tabular}{cc} 
Simulation cell size & Energy/electron \\
\hline $6 \times 6$ & $-0.04322(1)$ \\
$8 \times 6$ & $-0.04322(1)$ \\
$8 \times 8$ & $-0.04322(1)$ \\
$10 \times 8$ & $-0.04322(1)$ \\
$10 \times 10$ & $-0.04322(1)$ \\
$12 \times 10$ & $-0.04321(1)$ \\
\hline \hline
\end{tabular}

TABLE VIII. An estimate for the size of exchange contribution to the total energy: comparison of screened Hartree with screened Hartree-Fock (HF) for $r_{s}=20$ at $\nu=1 / 3$. For comparison, the unscreened results from Table IV are also shown here. Total energies (not including $\frac{1}{2} \hbar \omega_{c}$ ) are given in atomic units.

\begin{tabular}{lccc}
\hline \hline Bare Hartree & Bare HF & Screened Hartree & Screened HF \\
\hline-0.04722 & $-0.04762(9)$ & $-0.05035(1)$ & $-0.05035(1)$ \\
\hline \hline
\end{tabular}


TABLE IX. Ground state energies at $r_{s}=20$ for the Wigner solid versus the FQHE liquid from Ref. [7] in atomic units. We have taken out the term $\frac{1}{2} \hbar \omega_{c}$. For its value at respective filling factors, see Table V.

\begin{tabular}{ccc}
\hline \hline Filling factor $\nu$ & Wigner crystal & FQHE liquid \\
\hline $1 / 3$ & $-0.05073(1)$ & $-0.05090(1)$ \\
$1 / 5$ & $-0.05212(1)$ & $-0.05203(1)$ \\
$1 / 7$ & $-0.05291(1)$ & $-0.05265(1)$ \\
$1 / 9$ & $-0.05341(1)$ & $-0.05306(1)$ \\
\hline \hline
\end{tabular}




\section{FIGURES}

Fig. $1 E_{F M}-E_{A F M}$ (in $10^{-3}$ atomic units) for electrons in a square lattice. The line is a spline fit to guide the eye. The FM state has a lower energy.

Fig. $2 E_{F M}-E_{A F M}$ (in $10^{-3}$ atomic units) for electrons in a honeycomb lattice. The line is a spline fit to guide the eye. The AFM state has a lower energy.

Fig. $3 E_{F M}-E_{A F M}$ (in $10^{-3}$ atomic units) as a function of $r_{G}$, the width of the Gaussians in the one-particle orbitals, for electrons in the honeycomb lattice at $r_{s}=30$. The parameters $\mathrm{A}$ and $\mathrm{F}$ in the Jastrow factor are not altered with $r_{G}$.

Fig. $4 E_{F M}-E_{A F M}$ (in $10^{-3}$ atomic units) as a function of aspect ratio $a / b$ for the rectangular lattice at $r_{s}=30$. Gaussians in the one-particle orbitals are anisotropic (see text for details).

Fig. 5 Schematic illustration of Thouless and Li's [50] argument for the sign of the ringexchanges in a strong magnetic field. The underlying assumption is that the total energy increases with the number of nodes in the spatial part of the wavefunction. It is argued, in the text, that this assumption does not hold in the FQHE regime.

Fig. 6 Total energy (in effective atomic unit) as a function of $A_{p}$, the strength of the magneto-phonon correlation, for $\nu=1 / 3$ at $r_{s}=20$. The line is a spline fit. A constant $\frac{1}{2} \hbar \omega_{c}$ is subtracted.

Fig. 7 Kinetic energy (in atomic units) per electron vs $\beta$ for $\nu=1 / 3$ at $r_{s}=20$ in the effective atomic unit. The line is a spline fit.

Fig. 8 Interaction energy (in atomic units) per electron vs $\beta$ for $\nu=1 / 3$ at $r_{s}=20$ in the effective atomic unit. The line is a spline fit. 
Fig. $9 E^{\text {total }}-\frac{1}{2} \hbar \omega_{C}$ (per electron) vs $\beta$ for $\nu=1 / 5$ at $r_{s}=20$. Energies are in the effective atomic unit. Statistical noise are given by the size of the data points. The line is a spline fit.

Fig. $10 E^{\text {total }}-\frac{1}{2} \hbar \omega_{C}$ (per electron) vs $A$ for $\nu=1 / 3$ and $r_{s}=20$ calculated with $A_{p}=1$ and $\beta=1.17$. Energies are in the effective atomic unit. Statistical noise are given by the size of the data points. The line is a spline fit.

Fig. $11 \mathrm{WC}$ solid and the FQHE liquid energies at $\nu=1 / 3$ and $r_{s}=20$ in the effective atomic unit calculated from different wavefunctions. (The liquid remains the ground-state at $\left.r_{s}=20.\right)$ WC1: Hartree-Fock results with no Landau-level-mixing. WC2: Magneto-phonon correlated results with no Landau-level-mixing. WC3: Lowest possible energy without introducing the Landau-level-mixing Jastrow factor, but allows Landau-level-mixing by changing $\beta$. WC4: Lowest energy for the WC with all variational parameters optimized. FQHE1: FQHE liquid energy from the Laughlin wavefunction with no Landau-level-mixing [34. FQHE-2: FQHE liquid energy with Landau-level-mixing from Ref. [7].

Fig. 12 FQHE liquid and WC solid energies at $\nu=1 / 5$ at $r_{s}=20$ in the effective atomic unit calculated from different wavefunctions. (The solid has a lower energy at $r_{s}=20$.) The notations for the data points are identical to those in Fig. 11.

Fig. $13 E^{\text {total }}-\frac{1}{2} \hbar \omega_{C}$ of the WC at $r_{s}=20$ compared with those of the FQHE liquid. Energies are in effective atomic unit. Heavy line connecting the empty squares is the WC energy with no Landau-level-mixing but with magneto-phonon correlations. Heavy line connecting the filled squares is the WC energy with all correlation effects considered obtained in this work. Dotted line connecting the empty hexagons is the Laughlin state with no Landaulevel-mixing from Ref. [10]. Dotted line connecting the filled hexagons is the Laughlin state with Landau-level-mixing from Ref. [16]. 
Fig. 14 Schematic phase diagram of a $2 \mathrm{D}$ electron/hole system at $T=0$ and free of disorder. The $x$-axis is filling factor and the $y$-axis is the effective $r_{s}$. Several possible reentrant phase trsnsitions around the principles FQHE states are illustrated. We have assumed that at $\nu=1 / 7$ the ground-state is the Wigner crystal. 